\title{
不同催化体系下腈的水合反应研究进展
}

\author{
夏玉杰 ${ }^{a}$ 何丹丹 ${ }^{a}$ 伍婉卿 $*, a, b$ \\ ( ${ }^{a}$ 华南理工大学化学与化工学院 广东省功能分子工程重点实验室 广州 510640) \\ $\left({ }^{b}\right.$ 华南理工大学 发光材料与器件国家重点实验室 广州 510640)
}

\begin{abstract}
摘要 腈可用于构建新的碳一碳、碳一杂原子键, 所得产物丰富多样. 酰胺基团广泛存在于医药、农药和天然产物中, 此 外，酰胺还是有机合成反应中重要的中间体. 在目前报道的酰胺类化合物的合成方法中，腈的水合反应已成为学术界 和工业界最广泛使用的获得初级酰胺类化合物的方法之一. 早期腈的水合反应中通常涉及强酸、强碱的使用, 但在该 类反应体系下, 往往存在产率低及反应选择性差等问题, 且所得酰胺容易过度水解成羧酸. 为了克服这一局限实现腈 的高效水合, 且满足绿色化学的要求, 近年来, 不同的催化体系相继被开发, 如过渡金属配合物催化剂、金属阳离子催 化剂、金属纳米粒子催化剂、离子液体催化剂及其它类型催化剂. 对这些催化体系下腈的水合反应研究进行阐述和总 结, 并对该领域的发展前景进行了展望.
\end{abstract}

关键词 腈; 催化体系; 水合反应; 酰胺

\section{Recent Advances for Hydration Reaction of Nitriles in Different Catalytic Systems}

\author{
Xia, Yujie ${ }^{a}$ \\ He, Dandan ${ }^{a}$ \\ Wu, Wanqing ${ }^{*, a, b}$ \\ $\left({ }^{a}\right.$ Key Laboratory of Functional Molecular Engineering of Guangdong Province, School of Chemistry and Chemical \\ Engineering, South China University of Technology, Guangzhou 510640) \\ ( ${ }^{b}$ State Key Laboratory of Luminescent Materials and Devices, South China University of Technology, Guangzhou 510640)
}

\begin{abstract}
Nitriles can be used to construct new carbon-carbon and carbon-hetero bonds which result in diverse products. Among them, amide groups can be found in a large variety of drugs, pesticides, natural products and key intermediates in organic synthesis. Among all the methods reported for the synthesis of amides, hydration reaction of nitriles has become one of the most widely used methods to obtain primary amides in both academia and industry. Conventional nitrile hydration generally involves the use of strong acids and bases which would cause some problems such as low yields, poor reaction selectivity and over hydrolysis of products to carboxylic acids. To overcome these disadvantages, achieve the hydration of nitriles efficiently and meet the requirements of green chemistry, different catalytic systems have been successfully developed, including transition metal complex catalysts, metal cationic catalysts, metal nanoparticle catalysts, ionic liquid catalysts and other types of catalysts. The hydration reaction of nitriles in these catalytic systems is reviewed and summarized, and the development prospect of this field is prospected.
\end{abstract}

Keywords nitrile; catalytic system; hydration reaction; amide

酰胺基团是有机合成的重要官能团, 是肽、酶及蛋 白质的基本结构单元, 广泛存在于药物、农药和天然产 物中, 不仅如此, 酰胺作为重要的化工原料在润滑油、 塑料及清洁剂等方面均有应用 ${ }^{[1-3]}$. 酰胺通常由羧酸及 羧酸类衍生物如酸䣶、酯、卤化物与胺反应制备而得 ${ }^{[4-5]}$. 然而, 这些常见的方法往往存在着较多的局限性, 如利
用羧酸和胺反应制备酰胺, 需要添加化学计量甚至更多 的碱作为活化试剂，其在反应结束后成为副产物，原子 效率低, 不符合绿色化学理念.

腈作为一类分子中含有氰基的化合物, 既可以与亲 核试剂反应，也能够与亲电试剂作用构建新的碳一碳、 碳一杂原子键，如通过环化反应制备多环喹啉类化合

\footnotetext{
* Corresponding author. E-mail: cewuwq@scut.edu.cn

Received July 7, 2020; revised September 1, 2020; published online October 10, 2020.

Project supported by the National Natural Science Foundation of China (22071063).

国家自然科学基金(No. 22071063)资助项目.
} 
物 ${ }^{[6]}$ 及喹唑啉类化合物 ${ }^{[7]}$ 等. 由于具有独特的化学多样 性, 腈被广泛应用于科研和工业领域 ${ }^{[8]}$, 其中, 由腈水 合反应制备酰胺因其过程简单直接且原子效率高，成为 有机合成化学领域的研究热点. 但早期该类反应仍然存 在许多局限, 如需要强酸、强碱的参与, 条件苛刻, 产率 较低且酰胺往往会过度水解成羧酸 ${ }^{[9-10]}$, 反应结束后会 产生较多的盐, 同时还存在着环境和安全等问题, 不符 合可持续发展的绿色化学理念; 此外, 腈的水合反应通 常使用水分子作为亲核试剂, 但由于氰基的碳氮参键键 能高, 在化学反应中难以被活化, 因而利用水分子直接 对其进行亲核进攻是动力学缓慢的过程 ${ }^{[11]}$; 腈水合酶 因选择性高和反应过程所需能量较少, 受到研究工作者 的青睐, 但酶的分离成本高且容易受产物和原料的影 响, 如底物是 $\alpha$-差基腈时, 酶容易中毒而失去催化活性, 致使反应局限性较大 ${ }^{[12]}$. 为了解决这些难题, 几十年来 化学研究者致力于开发不同的催化剂催化腈的水合反 应，如金属配合物催化剂、金属阳离子催化剂、金属纳 米粒子催化剂及离子液体催化剂等, 由于氧基的反应惰 性，这些催化体系大多需要过渡金属如 $\mathrm{Pd} 、 \mathrm{Ru} 、 \mathrm{Rh}$ 、 $\mathrm{Cu} 、 \mathrm{Ir}$ 的参与. 在这些催化剂的作用下, 腈水合反应的 条件得以优化, 如避免强酸、强碱的使用, 在室温条件 进行, 短时间内完成转化, 减少有机溶剂的使用等. 为 了进一步了解腈水合反应的历程, 本文对近年来腈在不 同催化体系下的水合反应的研究做了简要的概述.

\section{1 金属配合物催化腈的水合反应}

早在 20 世纪末金属配合物催化剂就已经被开发出 来, 并被广泛应用于催化腈的水合反应. 经过几十年的 发展，金属配合物催化剂不断完善并趋于成熟. 该类催 化剂稳定性较好, 易于回收, 在反应过程中产物选择性 高, 官能团适用性广, 使腈能在相对温和的条件下转化 为酰胺.

1990 年, Kim 等 ${ }^{[13]}$ 利用三种不同的钴配合物催化剂 (图 1)在 $\mathrm{pH}=7$, 温度为 $40{ }^{\circ} \mathrm{C}$ 的条件下催化乙腈水合反 应成酰胺. 作者对三种催化剂的催化效果做了动力学实 验, 研究结果表明 2 的催化效果最佳, 催化速率为 $3.2 \times$ $10^{-6} \mathrm{~mol} \cdot \mathrm{L}^{-1} \cdot \mathrm{s}^{-1}$, 较 3 的催化速率高 20 倍, 而 $\mathbf{1}$ 在催化

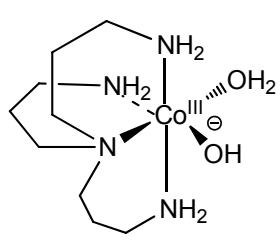

1

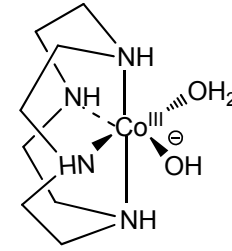

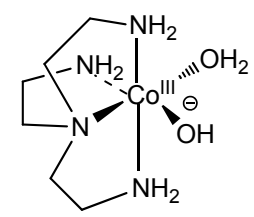

3
图 1 三种 $\mathrm{Co}(\mathrm{III})$ 配合物催化剂

Figure 1 Three kinds of Co(III)-complex catalysts
过程中会产生较多的杂质. 作者认为在催化过程中, 催 化剂 2 的金属中心与氰基中的氮原子配位得到配合物 4 , 增加了氰基中碳原子的亲电性，之后金属氢氧化物对碳 原子进行分子内亲核进攻, 从而得到产物酰胺(图 2).

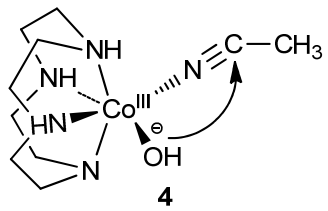

图 2 催化剂 2 与乙腈的配位方式

Figure 2 Coordination mode of catalyst 2 with acetonitrile

钓金属的价态范围广，从-2 到 +8 , 配位能力强, 可与多种配体以不同的方式配位形成不同类型的钉金 属配合物, 在催化腈的化学转化方面具有巨大的潜力. 早在 1974 年, Diamond 等 ${ }^{[14]}$ 就提出并运用钉配合物催化 剂催化腈反应生成酰胺，但在该催化体系下，钉催化剂 需要在氧化银、三氟乙酸以及锌录齐的共同作用下才能 完成腈的转化. 直到 Murahashi 等 ${ }^{[15]}$ 开发出催化剂 $\left[\mathrm{RuH}_{2}\left(\mathrm{PPh}_{3}\right)_{4}\right](5)$, 添加 2 equiv. 的水，以乙二醇二甲醚 为溶剂, 在 $120{ }^{\circ} \mathrm{C}$ 的条件下将部分烷基腈和芳基腈水 合成酰胺(Eq. 1), 产率高达 92\%. 在该催化剂的作用下, 无需添加其它金属催化剂和有机溶剂，自此，钉催化体 系才真正意义上被开发出来. 在反应过程中，作者认为 腈首先与催化剂未配位饱和的金属中心配位，完成氰基 的活化后，水分子对碳原子进行亲核进攻，然后经过异 构化过程脱去催化剂分子，最终得到产物酰胺(Scheme $1)$.
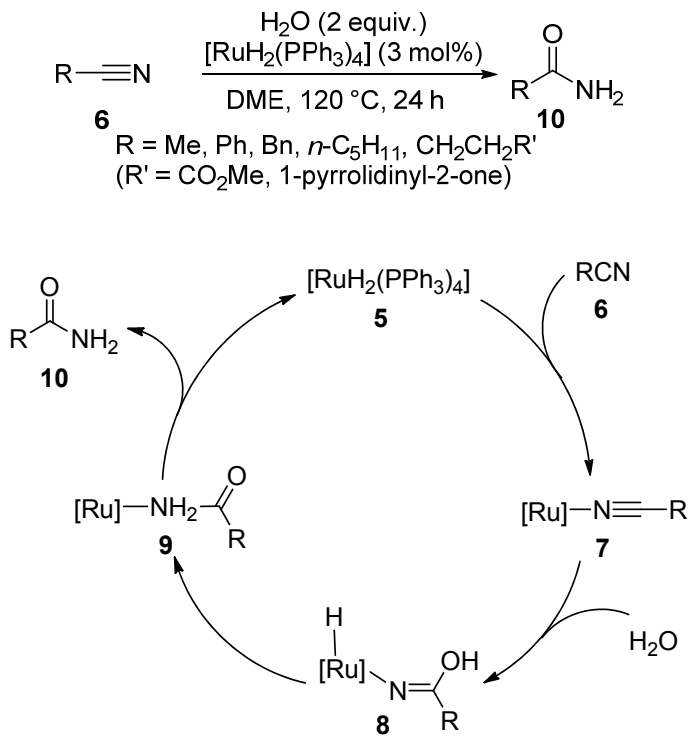

图式 $1\left[\mathrm{RuH}_{2}\left(\mathrm{PPh}_{3}\right)_{4}\right]$ 催化腈的水合反应机理

Scheme $1 \quad\left[\mathrm{RuH}_{2}\left(\mathrm{PPh}_{3}\right)_{4}\right]$-catalyzed reaction mechanism for hydration reactions of nitriles

与钴、钉配合物催化剂类似，在金属配合物催化剂 
催化腈转化反应的过程中, 催化剂金属中心与腈配位活 化氰基，再经过分子内或分子间的亲核进攻完成腈的转 化. 此外, 部分催化剂与底物分子之间能够形成氢键或 双氢键，不仅稳定了金属配合物，且在控制化学反应的 活性和区域选择性方面发挥重要作用, 这类催化剂被称 为 “双功能催化剂” , 即在反应过程中, 催化剂金属中 心作为路易斯酸活化腈, 配体作为路易斯碱活化亲核试 剂 ${ }^{[16]} .2003$ 年, Fung 等 ${ }^{[17]}$ 认为 $\left(\eta^{5}-\mathrm{C}_{9} \mathrm{H}_{7}\right)-\mathrm{Ru}(\mathrm{dppm}) \mathrm{H}(\mathbf{1 1})$ 可有效地将腈转化为酰胺, 而 $\left(\eta^{5}-\mathrm{C}_{9} \mathrm{H}_{7}\right)-\mathrm{Ru}(\mathrm{dppm}) \mathrm{Cl}$ (12)却没有催化活性, 密度泛函理论(DFT)计算结果也 验证了配体氢在反应过程中发挥重要作用. 在反应过程 中, 11 上的金属中心与氢配体和水分子形成双氢键 $\mathrm{Ru}-\mathrm{H} \cdots \mathrm{H}-\mathrm{OH}$ 得到中间体 15 , 由于双氢键的生成, 在 反应过程中提前得到过渡态 $15 \mathrm{a}$, 该过程是反应的决速 步骤, 所需活化能为 $15.9 \mathrm{~kJ} / \mathrm{mol}$, 与未形成双氢键的过 渡态 $15 \mathrm{~b}$ 相比, 活化能降低了 $72.4 \mathrm{~J} / \mathrm{mol}$, 再经过质子转 移过程得到配合物中间体 16 , 并最终生成产物酰胺 (Scheme 2).

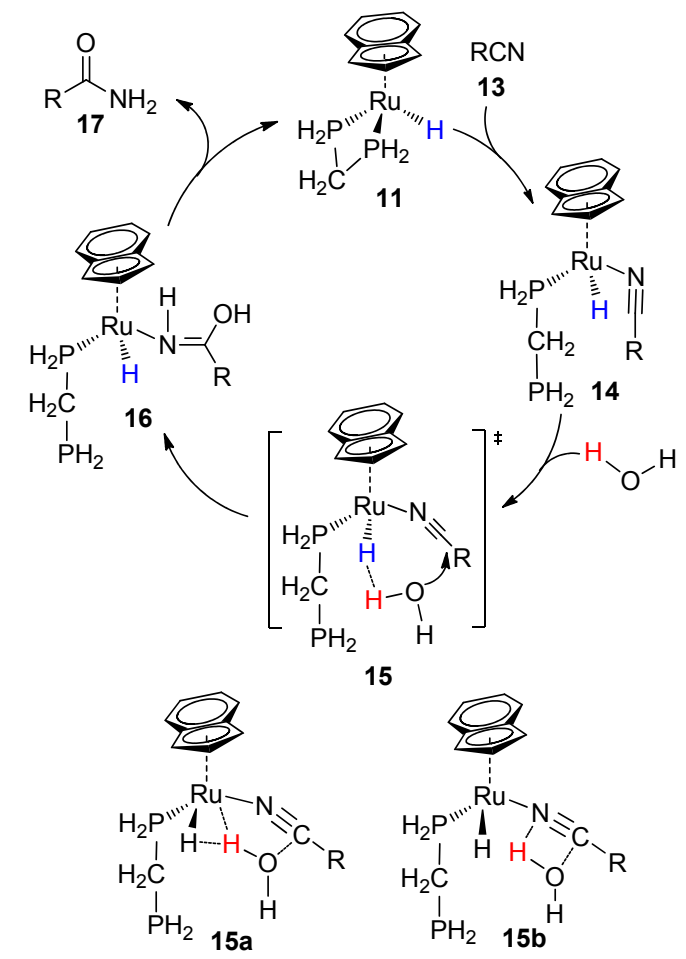

图式 $2\left(\eta^{5}-\mathrm{C}_{9} \mathrm{H}_{7}\right)-\mathrm{Ru}(\mathrm{dppm}) \mathrm{H}$ 催化腈的水合反应机理 Scheme $2 \quad\left(\eta^{5}-\mathrm{C}_{9} \mathrm{H}_{7}\right)-\mathrm{Ru}(\mathrm{dppm}) \mathrm{H}$-catalyzed reaction mechanism for hydration reactions of nitriles

在催化体系中, 催化剂配体与反应底物形成氢键时 能在大幅度提高催化效率的同时提高产率 ${ }^{[5]}$, 例如, 钉 金属配合物 $c i s-\left[\mathrm{Ru}(\mathrm{acac})_{2}\left(\mathrm{PPh}_{2} \mathrm{py}\right)_{2}\right]$ (18)在 $180{ }^{\circ} \mathrm{C}$ 的条 件下能催化大部分腈水合成酰胺. 在催化过程中, 18 的 金属中心首先与氧基配位, 配体中的氮原子与水分子中
的氢原子形成氢键，进一步活化水分子，促进水分子对 腈的亲核进攻. 而催化剂 $c i s$ - $\left[\mathrm{Ru}(\mathrm{acac})_{2}\left(\mathrm{PPh}_{3}\right)_{2}\right]$ (19)与水 分子之间不能形成氢键, 反应时间要远长于 18 , 这也证 明了氢键的形成对反应过程有着积极的影响. 研究表 明, 在体系中加入可形成氢键的配体时, 能提高本身不 能形成氢键催化剂的催化效率, 并且催化效率的提高与 催化剂上氢键受体配体的强度有关 ${ }^{[18-19]}$. 为了证实这一 理论，Knapp 等 ${ }^{[20]}$ 合成了五种不同膦配体的钓配合物催 化剂 $\left[\mathrm{Ru}\left(\eta^{6}\right.\right.$-arene) $\left.\mathrm{Cl}_{2}\left(\mathrm{PR}_{3}\right)\right]\left[\mathrm{R}=\mathrm{NMe}_{2}(\mathbf{2 0 a}), \mathrm{OMe}(\mathbf{2 0 b})\right.$, OEt (20c), Et (20d), ${ }^{i} \operatorname{Pr}$ (20e)], 并研究了它们催化腈(包 括 $\alpha$-羟基腈)水合反应生成酰胺的活性和机理. 作者提 出两种反应路径(Scheme 3): 分子内反应(path a) 和分子 间反应(path b). 作者在盐溶液中设计实验排除了路径 a, 认为该反应是通过分子间亲核进攻完成的，即腈与催化 剂金属中心配位后，水分子直接对氰基上的碳原子进行 亲核进攻得到产物. 从电子效应和空间位阻来看, 20a 与 20e 的催化效果相当, 20b 的催化效果应是最佳, 但实 验结果表明五种催化剂中催化剂 20a 的催化活性最高. 实验验证和 DFT 计算表明，催化剂 20a 配体中的氮原子 与水分子之间形成了较强的氢键(图 3), 氢键的产生促 进了水分子的脱质子过程, 进一步提高反应了效率, 从 而使得 20a 的催化效果优于其它催化剂.

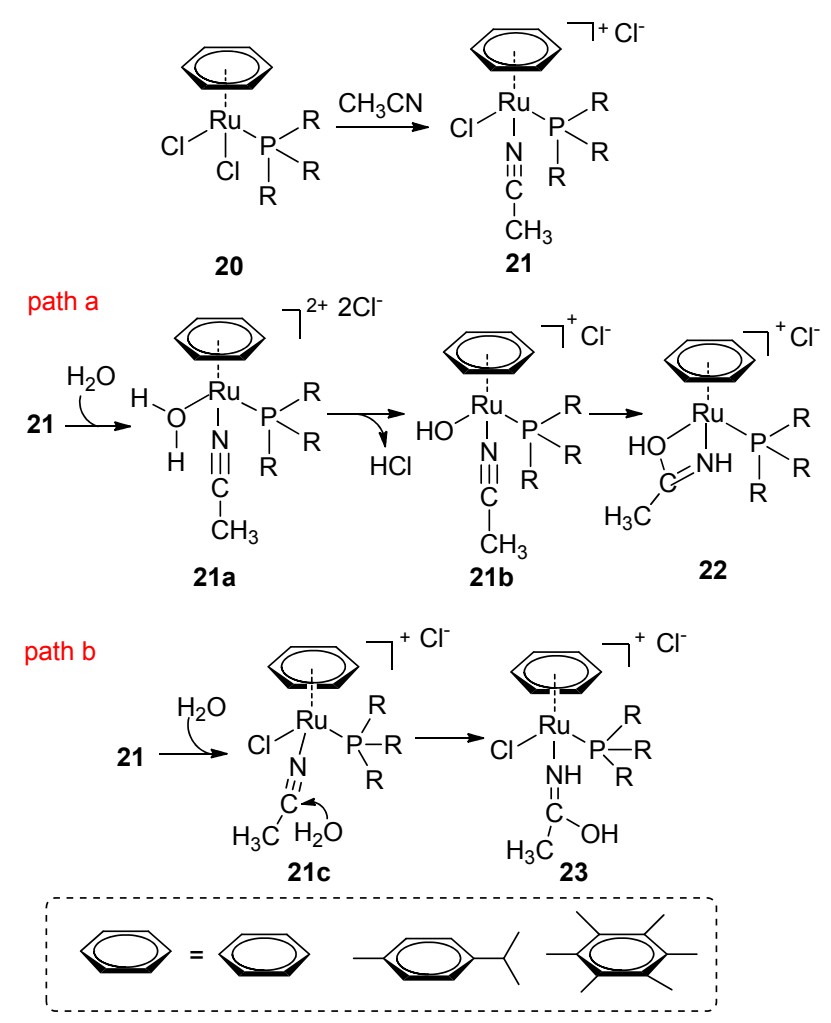

图式 $3\left[\mathrm{Ru}\left(\eta^{6}\right.\right.$-arene $\left.) \mathrm{Cl}_{2}\left(\mathrm{PR}_{3}\right)\right]$ 催化下腈的水合反应机理 Scheme $3 \quad\left[\mathrm{Ru}\left(\eta^{6}\right.\right.$-arene $\left.) \mathrm{Cl}_{2}\left(\mathrm{PR}_{3}\right)\right]$-catalyzed reaction mechanisms for hydration reactions of nitriles 


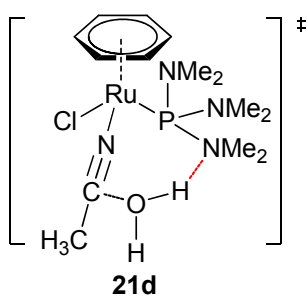

图 3 催化剂与水分子之间形成的氢键

Figure 3 Hydrogen-bond between the catalyst and the water molecule

$\mathrm{P}\left(\mathrm{NR}_{2}\right)_{3}$ 配体在腈的水合反应过程中可以与水分子 形成氢键, 促进亲核进攻的过程. 与 20a 的催化机制相 似, 2014 年, Tomás-Mendivil 等 ${ }^{[21]}$ 合成了一系列铑金属 配合物 $\left[\mathrm{RhCl}(\mathrm{COD})\left(\mathrm{P}\left(\mathrm{NR}_{2}\right)_{3}\right)\right]$ (24), 其中 24a (图 4)在没 有任何添加剂存在的条件下可使大部分芳基腈、杂芳基 腈和烷基腈在水溶液中高效转化(Eq. 2). 在催化过程中, 24a 的金属中心与氰基配位, 同时配体的氮原子与水中 的氢原子形成氢键得到中间体 26, 增强了水分子的亲 核性，提高了产物的选择性和收率(图 5).

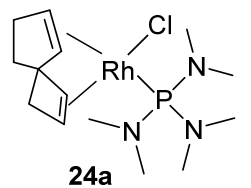

图 $4\left[\mathrm{RhCl}(\mathrm{COD})\left\{\mathrm{P}\left(\mathrm{NMe}_{2}\right)_{3}\right\}\right]$ 的结构

Figure 4 Structure of $\left[\mathrm{RhCl}(\mathrm{COD})\left\{\mathrm{P}\left(\mathrm{NMe}_{2}\right)_{3}\right\}\right]$
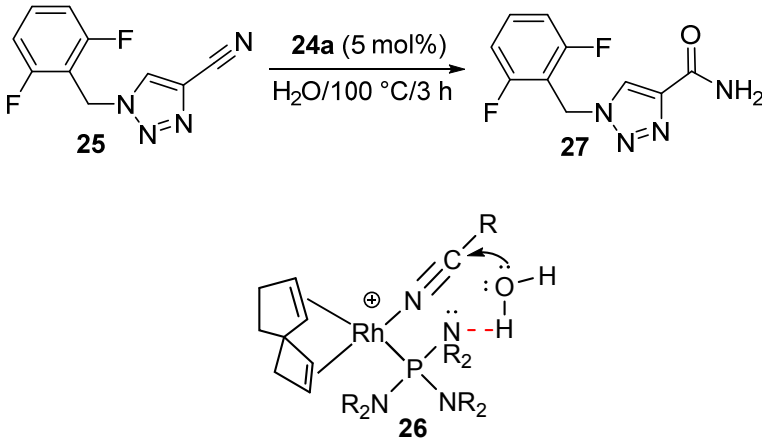

图 5 催化剂 $24 \mathrm{a}$ 与水分子之间形成的氢键

Figure 5 Hydrogen-bond between the catalyst 24a and the water molecule

同年，Tomás-Mendivil 等 ${ }^{[22]}$ 将 Pregosin 等 ${ }^{[23-24]}$ 合成 的钌金属配合物 29 和 30 作为催化剂, 催化腈的水合反 应 (Scheme 4). 该催化剂的金属中心连接一分子 $\mathrm{PPh}_{2} \mathrm{OH} 、 \mathrm{Cl}$ 或 OTf 基团, 在反应过程中催化剂与三分 子腈配位, $\mathrm{PPh}_{2} \mathrm{OH}$ 上的 $\mathrm{OH}$ 对腈进行亲核进攻, 形成中 间体 32, 之后在水的作用下得到产物酰胺(Scheme 5).

钉金属价格低廉, 配位能力强, 因此越来越多的钉 配合物催化剂被开发出来, 如 $\left[\mathrm{Ru}(\mathrm{Tp})\left(\mathrm{PPh}_{3}\right)\left(\mathrm{H}_{2} \mathrm{O}\right)\{\mathrm{NH}-\right.$
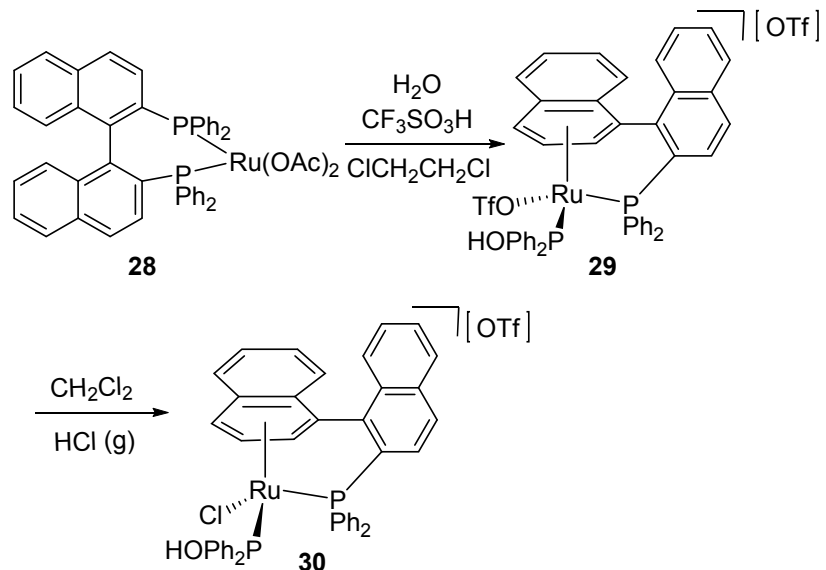

图式 4 催化剂 29 和 30 的合成

Scheme 4 Synthesis of the catalyst 29 and $\mathbf{3 0}$

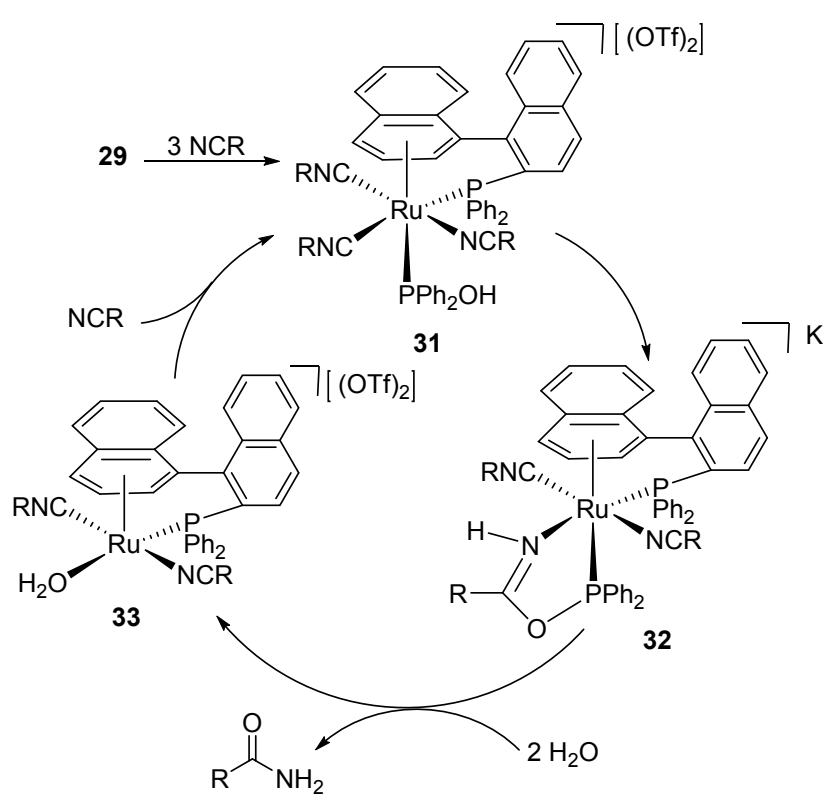

图式 5 配合物 29 催化的腈的水合反应机理

Scheme 5 Complex 29-catalyzed reaction mechanism for the hydration reactions of nitriles

$\mathrm{C}(=\mathrm{O}) \mathrm{Me}\}]^{[25]}(\mathbf{3 4}) 、\left[\mathrm{RuCl}_{2}\left(\eta^{3}: \eta^{3}-\mathrm{C}_{10} \mathrm{H}_{16}\right)\left\{\mathrm{PR}_{2}(\mathrm{OH})\right\}\right]^{[26]}$ (35)等均能在较温和的条件下实现了腈的水合反应，然 而大多数金属配合物催化剂对 $\alpha$-羟基腈的转化效果不 佳, 反应中产生的 $\mathrm{HCN}$ 会使催化剂中毒失去活性, 进 而影响 $\alpha$-羟基腈的水合过程. 而铂金属配合物催化剂的 开发使 $\alpha$-羟基腈有望在温和的条件下完成水合反应. 1995 年, Parkins 等 ${ }^{[27]}$ 设计出一种铂配合物催化剂, $\mathrm{HPt}\left[\left(\mathrm{PMe}_{2} \mathrm{O}\right)_{2} \mathrm{H}\right]\left(\mathrm{PMe}_{2} \mathrm{OH}\right)$ (Parkins 催化剂) (36), 该催 化剂可用于催化腈的水合反应. Parkins 催化剂的金属中 心上连接三个 $\mathrm{Me}_{2} \mathrm{HP}=\mathrm{O}$, 其中两个 $\mathrm{Me}_{2} \mathrm{HP}=\mathrm{O}$ 以氢 键的方式连接在一起，第三个 $\mathrm{Me}_{2} \mathrm{HP}=\mathrm{O}$ 基团提供一个 游离的差基作为亲核试剂，在金属中心与腈配位后通过 分子间亲核进攻生成中间体 37, 最终得到产物酰胺(图 
$6)$.

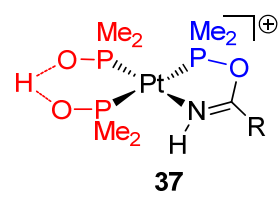

图 6 中间体 37 的结构

Figure 6 Structure of intermediate 37

Grubbs 等 ${ }^{[28]}$ 认为 Parkins 催化剂上以氢键相连的 $\mathrm{Me}_{2} \mathrm{HP}=\mathrm{O}$ 起到了双齿配体的作用, 这种富电子的双齿 配体稳定了整个催化剂, 增强了 $\mathrm{Me}_{2} \mathrm{HP}=\mathrm{O}$ 的亲核性, 并且与缺电子的二甲基氧化膦形成一种 “ donor-acceptor” 型催化剂, 有利于腈的活化. 在该催化体系下, 虽 然腈的转化效果较好, 但 $\alpha$-差基腈的反应效果不佳, 底 物范围窄. 为了解决这个问题, Grubbs 等 ${ }^{[28]}$ 设计了 3 种 新型的 “donor-acceptor” 型铂配合物催化剂 $38 \mathbf{a} 、 38 b$ 和 38c (图 7), 在较低的温度下实现了腈和 $\alpha$-羟基腈的 水合反应 $\left(\right.$ Scheme 6). 当反应温度为 $40{ }^{\circ} \mathrm{C}$ 、反应时间 $40 \mathrm{~h}$, 在 38a 的催化下, 乙腈水合反应的转化数(TON)值 高达 12712 , 远远高于 $36(\mathrm{TON}=5700)$. 含供电子能力 更强的 $38 \mathrm{a}$ 最短在 $10 \mathrm{~min}$ 内可完成底物的转化. 据报道, 这是第一个在室温条件下即可完成部分 $\alpha$-差基腈的水 合反应的催化剂. 作者在对三种催化剂进行 X 射线表征 时发现, 催化剂 Pt 金属中心与配体几乎形成了一个平 面配位结构, 其中 $\mathrm{P}^{\prime}-\mathrm{Pt}-\mathrm{P}\left(\mathrm{Me}_{2} \mathrm{OH}\right)$ 的角度分别为 $167.6^{\circ}$ 、 $166.5^{\circ}$ 和 $167.1^{\circ}$ (图 8), 这种接近线性的结构增强了 $\mathrm{PMe}_{2} \mathrm{OH}$ 中 $\mathrm{OH}$ 的亲核性, 从而提高了产率和选择性.

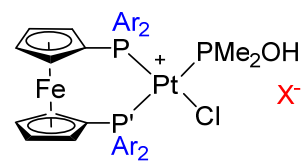

38a: $\mathrm{Ar}=\mathrm{Ph}, \mathrm{X}=\mathrm{OTf}$

38b: $\mathrm{Ar}=\mathrm{Ph}, \mathrm{X}=\mathrm{BF}_{4}$

38c: Ar $=5$-Me-2-furyl, $X=$ OTf

图 7 三种 “donor-acceptor” 型催化剂

Figure 7 Three "donor-acceptor" catalysts

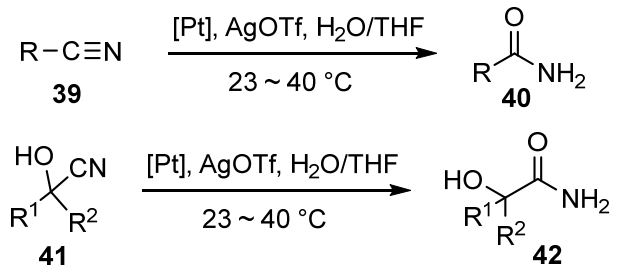

图式 6 腈和 $\alpha$-差基腈的水合反应

Scheme 6 Hydration reactions of nitriles and $\alpha$-hydroxynitriles

在金属配合物催化剂的作用下，腈经过水合反应生

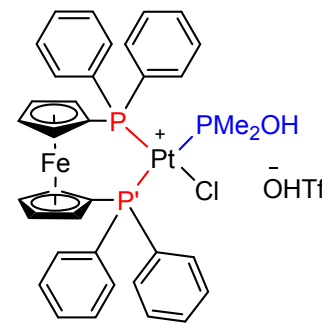

$38 a$

$$
\begin{aligned}
& \text { P'-Pt-P }\left(\mathrm{Me}_{2} \mathrm{OH}\right)=167.5^{\circ} \\
& \text { P-Pt-P' }=99.1^{\circ}
\end{aligned}
$$
$\mathrm{Cl}-\mathrm{Pt}-\mathrm{P}\left(\mathrm{Me}_{2} \mathrm{OH}\right)=82.5^{\circ}$

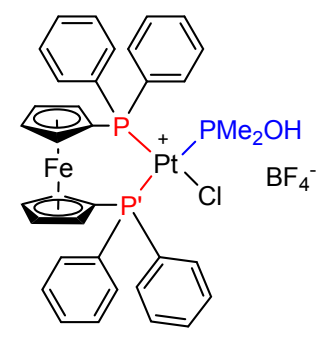

$38 \mathrm{~b}$

$\mathrm{P}^{\prime}-\mathrm{Pt}-\mathrm{P}\left(\mathrm{Me}_{2} \mathrm{OH}\right)=166.5^{\circ}$ $\mathrm{P}-\mathrm{Pt}-\mathrm{P}^{\prime}=100.0^{\circ}$ $\mathrm{Cl}-\mathrm{Pt}-\mathrm{P}\left(\mathrm{Me}_{2} \mathrm{OH}\right)=82.7^{\circ}$

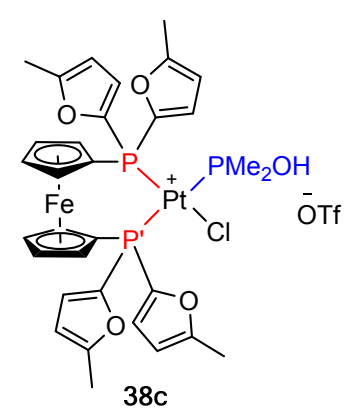

$\mathrm{P}^{\prime}-\mathrm{Pt}-\mathrm{P}\left(\mathrm{Me}_{2} \mathrm{OH}\right)=167.1^{\circ}$ P-Pt-P' $=98.5^{\circ}$ $\mathrm{Cl}-\mathrm{Pt}-\mathrm{P}\left(\mathrm{Me}_{2} \mathrm{OH}\right)=83.3^{\circ}$

图 $838 \mathrm{a}, 38 \mathrm{~b}$ 和 $38 \mathrm{c}$ 中的 $\mathrm{P}^{\prime}-\mathrm{Pt}-\mathrm{P}\left(\mathrm{Me}_{2} \mathrm{OH}\right)$ 角

Figure $8 \mathrm{P}^{\prime}-\mathrm{Pt}-\mathrm{P}\left(\mathrm{Me}_{2} \mathrm{OH}\right)$ angles in 38a, 38b and 38c

成酰胺, 酰胺也可经过 $N$-甲基化过程进一步转化为具 有药用价值的 $N$-甲基化酰胺. 2019 年, Paul 等 ${ }^{[29]}$ 报道了 腈和甲醇在钴配合物催化剂 $\mathrm{CoBr}_{2} / \mathrm{PP}_{3}$ 的作用下经过水 合反应、 $N$-甲基化反应两个过程直接得到 $N$-甲基化酰胺 (Eq. 3). 在对反应进行速率测试时, 作者发现电子效应 对反应速率影响较大, 腈水合反应速率远远大于 $N$-甲 基化反应的速率，当腈的间位或对位存在吸电子基团 时, 这种差异更为明显. 但取代基离酰胺键较远，电子 效应的影响不容易观察. 为了探究电子效应对反应过程 的影响，作者对其进行 DFT 计算，计算结果显示，当腈 的对位上分别存在 $\mathrm{CF}_{3} 、 \mathrm{H} 、 \mathrm{OMe}$ 时, 反应所需的活化 能分别为 $0.15 、 8.79 、 10.92 \mathrm{~kJ}^{\circ} \mathrm{mol}^{-1}$, 这一结果表明腈 的对位上存在吸电子基时，参与反应所需的活化能更 低，反应效果更好。通过机理验证，作者认为该反应主 要经历两个循环, 即腈的水合过程和 $N$-甲基化过程. 在 水合过程中，氧基与催化剂金属中心配位形成 45, 水对 45 进行亲核进攻后, 经过互变异构过程得到酰胺; $N$-甲 基化过程中，甲醇在催化剂和碱的作用下形成 $\mathbf{5 0 , 5 0}$ 经 过 $\beta-\mathrm{H}$ 消除生成 $\mathrm{Co}-\mathrm{H}$ 物种和甲醛, 酰胺和甲醛经过缩 合反应得到产物(Scheme 7). 腈经过水合反应生成酰胺, 再经过 $N$-甲基化得到 $N$-甲基化酰胺，这一过程不仅实 现了腈的转化利用, 也进一步扩大了酰胺的应用范围. 


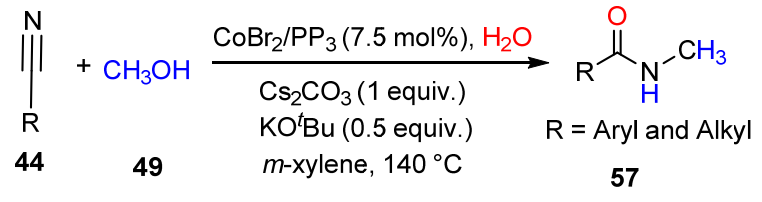

Nitrile hydration
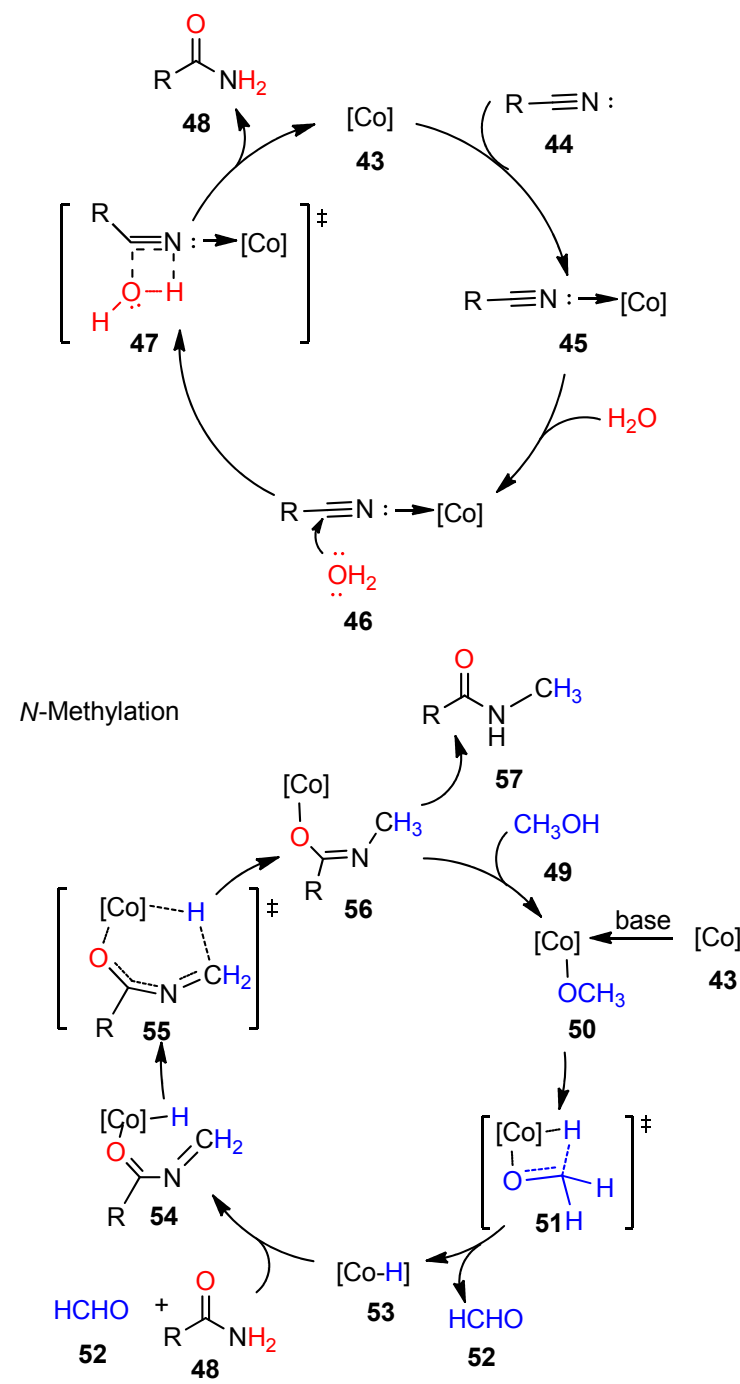

图式 $7 \mathrm{CoBr}_{2} / \mathrm{PP}_{3}$ 催化的腈的水合反应和酰胺的 $N$-甲基化反 应机理

Scheme $7 \mathrm{CoBr}_{2} / \mathrm{PP}_{3}$-catalyzed reaction mechanisms for hydration reactions of nitriles and N-Methylation reactions of amides

金属配合物催化剂发展较快, $[\mathrm{Mo}]^{[30]} 、[\mathrm{Ir}]^{[31]}$ 、

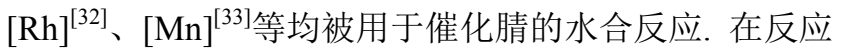
过程中，大多利用催化剂金属中心与氰基配位，从而活 化氰基的碳氮参键，最后通过分子内或分子间的亲核进 攻生成产物酰胺. 此外, 催化剂的配体之间相互作用或 与底物作用形成氢键或双氢键等提高转化效率和产率. 与强酸、强碱相比，在金属配合物存在的催化体系下， 催化剂的使用量和有机溶剂的添加量得到减少, 且能有 效控制酰胺进一步水解成羧酸, 减少了副产物的生成,
同时能兼容含有不同官能团的底物，使腈在较温和的条 件下高效地转化为酰胺. 但在催化过程中依旧存在着底 物范围受限的问题，芳基腈、杂芳基腈在一定条件下能 实现高效转化，大部分的烷基腈和 $\alpha$-羟基腈并不能高效 地转化, 这也成为化学研究者今后工作的焦点和难点.

\section{2 金属阳离子催化腈的水合反应}

近年来，大量的金属阳离子催化剂如 $[\mathrm{Pd}] 、[\mathrm{Cu}] 、[\mathrm{Ir}]$ 等被用来催化腈的转化以构建新的碳一碳、碳一杂原子键. 2009 年, $\mathrm{Kim}_{\text {等 }}{ }^{[34]}$ 首次用 $\mathrm{Pd}(\mathrm{OAc})_{2}$ 在甲苯溶液中催化腈 与乙醛肜反应生成酰胺, 乙醛肜作为亲核试剂对腈的进 攻，能够使反应过程更加稳定且产物较容易分离. 在该 报道中，大部分芳基腈都能有效转化为酰胺，产率良好， 最高可达 $93 \%$ ，反应过程经过两个主要过程(Scheme 8): 氰基与吸电子能力较强的催化剂金属中心配位，提高氧 基中碳原子的亲电性; 乙醛肜中的氧原子对腈亲核进攻 形成中间体 61，中间体 61 随后分解成一分子酰胺和一 分子乙腈. 虽然该体系对芳基腈有较好的催化效果，但 对烷基腈和杂芳基腈的兼容性较差，且反应过程伴随着 副产物乙腈的生成, 催化剂用量达到 $10 \mathrm{~mol} \%$. 因此, 如何在提高产率的同时，使用廉价的金属阳离子催化 剂，减少有机溶剂的使用和副产物的生成，让反应过程 更加环境友好仍是一个挑战.

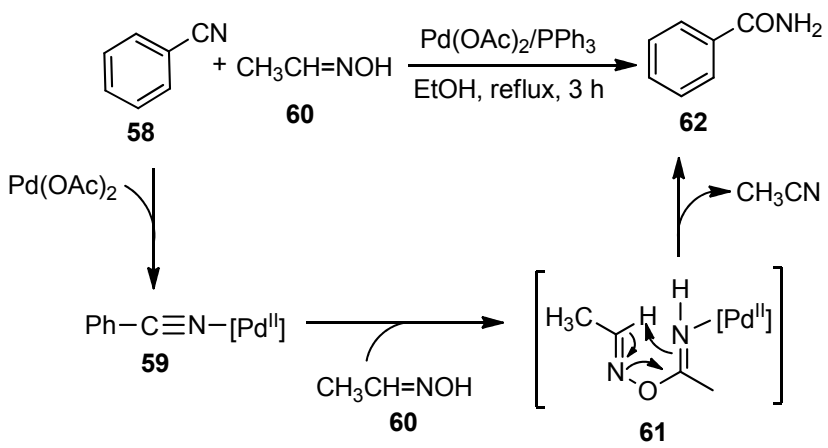

图式 $8 \mathrm{Pd}(\mathrm{OAc})_{2}$ 催化的腈的水合反应机理

Scheme $8 \mathrm{Pd}(\mathrm{OAc})_{2}$-catalyzed reaction mechanism for hydration reactions of nitriles

2010 年, 该课题组 ${ }^{[35]}$ 用 $\mathrm{InCl}_{3}$ 催化腈与乙醛肜反应 生成酰胺，与 $\mathrm{Pd}(\mathrm{OAc})_{2}$ 相比，在增加体系适用性的同时 不会使乙醛肜失活，催化剂含量降为 $5 \mathrm{~mol} \%$ ，在该催化 条件下，含有吸电子基团和供电子基团的芳基腈均可获 得较高的转化率，且部分烯基腈、烷基腈及杂芳基腈也 能取得较好的反应效果, 产率高达 $99 \%$. 与 $\mathrm{Pd}(\mathrm{OAc})_{2}$ 催 化腈与乙醛肜反应机理类似, 氰基首先与催化剂金属中 心配位，然后接受乙醛肜的亲核进攻形成中间体 64，再 分解为一分子酰胺和一分子乙腈(Scheme 9). 


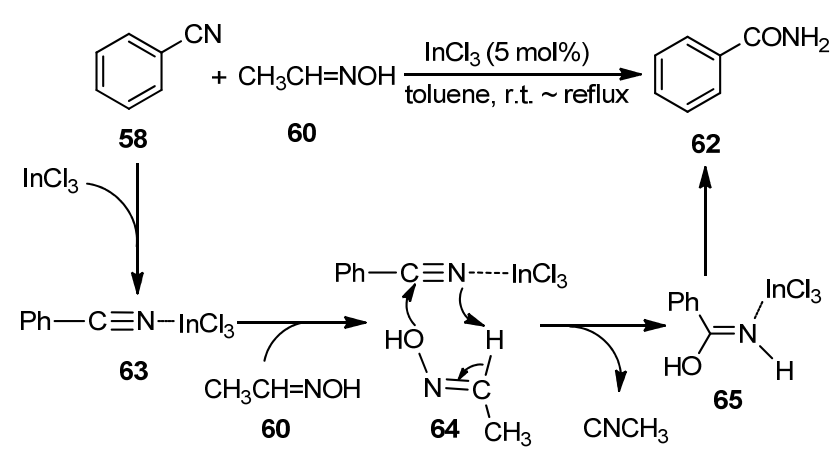

图式 $9 \mathrm{InCl}_{3}$ 催化的腈的水合反应机理

Scheme $9 \mathrm{InCl}_{3}$-catalyzed reaction mechanism for hydration reactions of nitriles

与 $\mathrm{Kim}$ 等报道不同的是, 2012 年, 陆明等 ${ }^{[36]}$ 以水为 溶剂、 $\mathrm{CuO}$ 为催化剂, 在回流的条件下促进腈与乙醛肜 反应生成酰胺. 该催化体系不仅避免了乙醇及甲苯等有 机溶剂的使用，乙醛肟的添加量也减少为 1.5 equiv. 该 催化体系对含氮杂环腈的适用性更广, 可连接不同取代 基, 反应效果较好. 与 $[\mathrm{Pd}] 、[\mathrm{Ir}]$ 的催化机制类似, 反应过 程中, 氭基与 $\mathrm{CuO}$ 配位后, 乙醛肜对腈进行亲核进攻得 到一分子酰胺与一分子乙腈(Scheme 10).

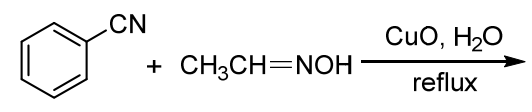<smiles>NC(=O)c1ccccc1</smiles>

58

60

62

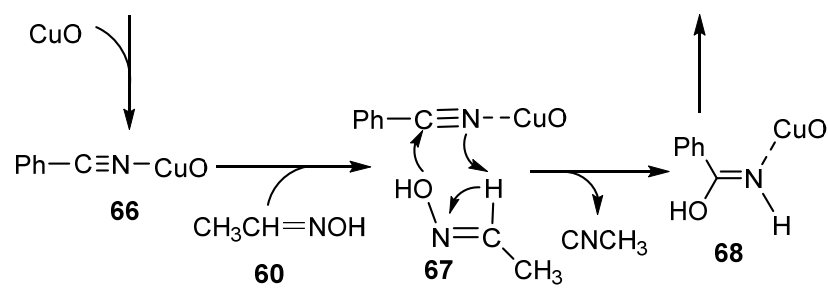

图式 $10 \mathrm{CuO}$ 催化的腈的水合反应机理

Scheme 10 CuO-catalyzed reaction mechanism for hydration reactions of nitriles

同年, 该课题组 ${ }^{[37]}$ 探究了不同金属盐对腈水合反 应催化活性的影响, 研究发现金属阳离子半径较小的 $\mathrm{NiCl}_{2} \cdot 6 \mathrm{H}_{2} \mathrm{O}$ 具有较高的催化活性. 含有吸电子取代基的 腈和杂芳基腈在室温条件下可高效地转化成相应的酰 胺. 作者提出腈类化合物如芳基腈和烷基腈的反应机理 与文献报道类似，即催化剂金属中心与氰基配位，乙醛 肜对其进行亲核进攻, 得到产物酰胺和一分子乙腈; 而 对于杂芳基腈, 在反应过程中杂原子与氰基同时与催化 剂配位, 形成一个五元环金属配合物 70.70 的形成有效 活化了氧基, 增强了㲵基上碳原子的亲电性, 促进反应 的进行(Scheme 11). 2013 年, 该课题组 ${ }^{[38]}$ 用 $\mathrm{Na}_{2} \mathrm{MoO}_{4}$ • $2 \mathrm{H}_{2} \mathrm{O}$ 作为腈水合反应催化剂, 乙醛肜为亲核试剂, 在
回流条件下芳香腈、杂芳基腈及烷基腈均能较好地转化 成相应的酰胺.

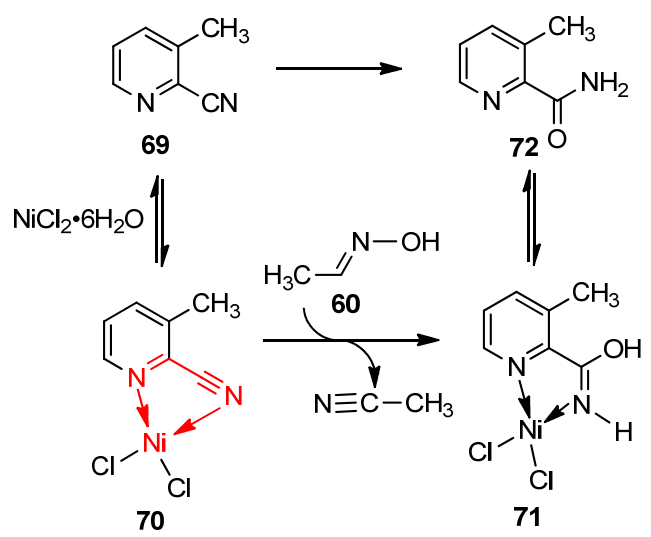

图式 $11 \mathrm{NiCl}_{2} \cdot \mathrm{H}_{2} \mathrm{O}$ 催化的腈的水合反应机理 Scheme $11 \mathrm{NiCl}_{2} \cdot \mathrm{H}_{2} \mathrm{O}$-catalyzed reaction mechanism for hydration reactions of nitriles

2017 年, Sanz 等 ${ }^{[39]}$ 在不添加肜和羟胺的前提下, 以 $\mathrm{Pd}(\mathrm{OAc})_{2}$ 为催化剂, 水为溶剂, $2,2^{\prime}$-联吡啶为配体, $70{ }^{\circ} \mathrm{C}$ 为反应最佳温度, 实现了部分烷基腈、芳基腈和杂 芳基腈的水合反应(Eq. 4). 在体系中加入 1,4-二氧六环 作为辅助溶剂促进芳基腈的溶解. 作者提出三种可能的 机理(Scheme 12). 三种路径中腈均先与 $\mathrm{Pd}(\mathrm{OAc})_{2}$ 进行 配位得到 74. 在路径(a)中, 74 直接受到水的进攻, 经过 重排后得到产物酰胺; 路径(b)和(c)中, 得到 74 后, Pd-

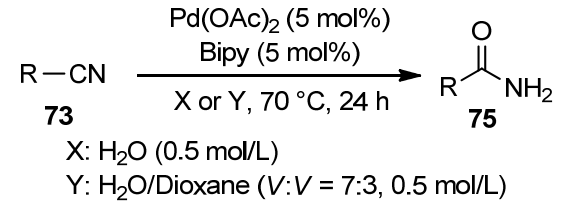
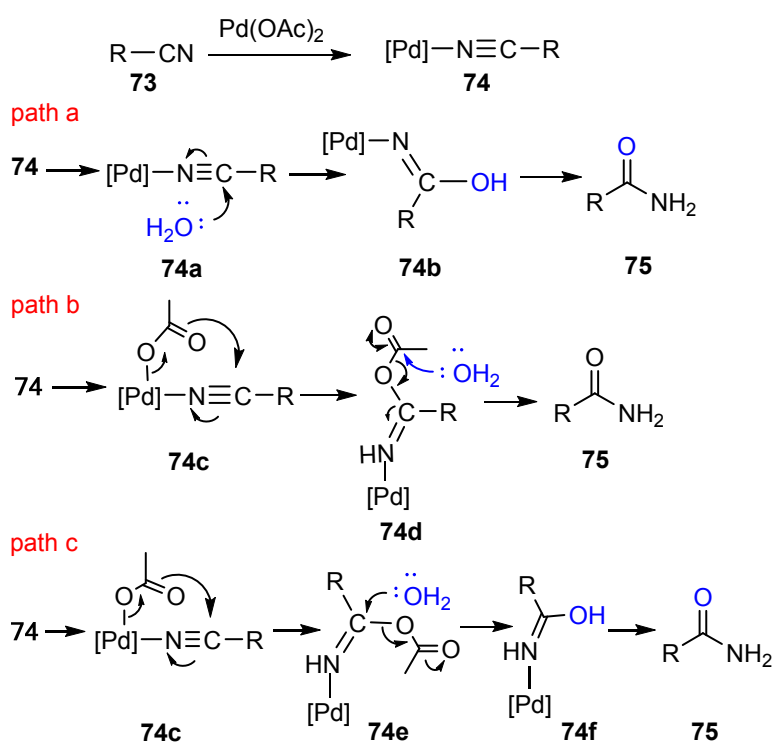

图式 12 三种可能的反应途径

Scheme 12 Three possible reaction mechanisms 
$(\mathrm{OAc})_{2}$ 本身向内传递氧, 形成中间体 74c, 随后水分别 进攻羧酸根中的碳原子和腈中的碳原子得到酰胺. 为了 了解具体的反应途径, 作者对水中的氧原子进行同位素 标记, 发现产物酰胺中的氧原子来自水分子, 进而排除 了路径(b); 催化剂 $\mathrm{PdCl}_{2}$ 和 $\mathrm{Pd}(\mathrm{OAc})_{2}$ 相比, 相应反应产 率大大降低, 说明醋酸根离子在催化过程中必不可少, 验证了反应路径(c).

金属阳离子催化腈的水合反应中大多使用水、乙醛 肟作为亲核试剂, 2019 年, $\mathrm{Kanda}$ 等 $^{[40]}$ 用 $\mathrm{Pd}\left(\mathrm{NO}_{3}\right)_{2}$ 催化 $\alpha$-差基腈与酰胺反应生成 $\alpha$-差基酰胺(Eq. 5), 该体系中 酰胺代替水或乙醛肜作为亲核试剂参与反应. $\alpha$-羟 基腈在 $50{ }^{\circ} \mathrm{C} 、 10 \mathrm{~min}$ 内高效地转化为相应的酰胺, 与 以往金属催化 $\alpha$-羟基腈的转化有所不同，该方法可有效 催化 $\alpha, \alpha$-二芳基取代羟基腈(即酮衍生的 $\alpha$-差基腈)的转 化, 且催化剂用量为 $2 \sim 5 \mathrm{~mol} \%$. 该反应经历四个过程 (Scheme 13): 首先 $\alpha$-羟基腈取代了催化剂中的一个配 体与金属中心配位形成阳离子钯物种, 其次酰胺取代催 化剂中的另一个配体并进攻氰基, 最终解离成产物酰胺 并释放出一分子乙腈.
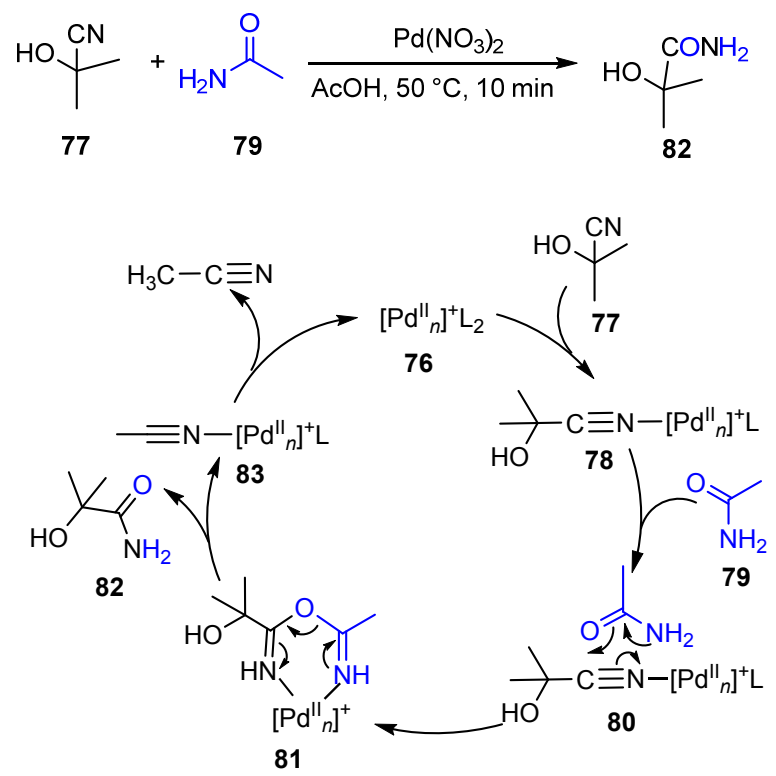

图式 $13 \mathrm{Pd}\left(\mathrm{NO}_{3}\right)_{2}$ 催化的 $\alpha$-差弪基腈的水合反应机理

Scheme $13 \mathrm{Pd}\left(\mathrm{NO}_{3}\right)_{2}$-catalyzed reaction mechanism for hydration reactions of $\alpha$-hydroxynitriles

金属阳离子催化剂在反应过程中表现出较高的催 化活性, 底物的官能团适用性广, 产物选择性和产率较 高, 且催化剂大多商业可得, 避免了繁琐的预处理过程. 催化机制与金属配合物催化剂类似, 在反应过程中催化 剂金属中心与氰基配位，达到活化氰基的目的; 最后， 亲核试剂对氰基上的碳原子进行分子间亲核进攻得到 产物酰胺. 但多数条件下使用的为贵金属催化剂, 价格
昂贵，且在反应过程中容易失活，不能重复利用. 因此, 在提高产率的同时, 减少催化剂的添加量, 使用价格低 廉的金属阳离子催化剂是腈水合反应的一个重要目标.

\section{3 金属纳米粒子催化腈的水合反应}

近年来, 金属配合物催化体系、金属阳离子催化体 系发展迅速，使不同类型的腈如芳基腈、烷基腈和杂芳 基腈能在相对温和的条件下以较高的收率转化成相应 的酰胺. 然而这些均相催化剂在催化过程中也存在反应 底物受限的问题，例如当反应底物为 $\alpha$-羟基腈时， $\alpha$-差弪 基腈分解成酮和氰化氢，其中氰化氢会毒害催化剂，从 而使 $\alpha$-差基腈的水合效果差. 金属纳米粒子催化剂介于 均相和非均相催化剂之间, 颗粒尺寸大小均匀的纳米粒 子催化剂能有效增加催化活性位点的暴露面积, 有利于 反应底物与催化剂的充分接触, 同时, 在反应结束后催 化剂能从体系中分离重复使用. 独特的性质使金属纳米 粒子催化剂如 $\mathrm{Pd}^{[41]} 、 \mathrm{Ag}^{[42-45]} 、 \mathrm{Ni}^{[46]}$ 及 $\mathrm{Ru}^{[47]}$ 等能催化不 同类型的反应 ${ }^{[48]}$ 包括氢化、氧化和光催化反应等，亦能 催化腈的水合反应，同时在一定程度上解决了均相体系 内过渡金属催化剂存在的局限性，拓展了腈水合反应的 底物范围.

研究表明，当金属表面吸附氧原子时，对水的分解 能力远远强于纯净的金属表面，且金属表面上的氧原子 可作为助催化剂在低温下促进各种有机反应 ${ }^{[49-51]}$. 为 此, 2012 年, Shimizu 等 ${ }^{[52]}$ 用一系列 Pd NPs 作为非均相 催化剂催化腈水合反应生成酰胺. 其中 $\mathrm{Pd} / \mathrm{C}-500 \mathrm{H} 84$ 由 $\mathrm{Pd}-\mathrm{C}$ 材料在 $500{ }^{\circ} \mathrm{C}$ 条件下, 在流动的 $\mathrm{H}_{2}$ 中反应 $10 \mathrm{~min}$ 并在氦气中冷却至室温制备而得, 将 84 在室温条件下 置于空气中吸附氧气 $\left(\mathrm{O}_{\mathrm{ad}}\right)$ 得到 $\mathrm{Pd} / \mathrm{C}-500$ Hox 85. 少量 的 85 , 在较低的反应温度下即能使活性较低的烷基腈、 高配位的吡啶腈、芳基腈转化成相应的酰胺, 而表面没 有 $\mathrm{O}_{\mathrm{ad}}$ 的 84 几乎不具有催化活性. 且随着 $\mathrm{O}_{\mathrm{ad}}$ 的增加, 催化剂中 $\mathrm{Pd}^{0}$ 位点减少, 转化频率(TOF)值降低, 这意味 着 $\mathrm{O}_{\mathrm{ad}}$ 和 $\mathrm{Pd}^{0}$ 均为反应的活性位点. 在反应过程中, $\mathrm{O}_{\mathrm{ad}}$ 作为布朗斯特碱与水分子中的氢原子生成较强的氢键, 促进水的解离, 而捕获质子, 在 $\mathrm{Pd}$ 的表面形成 $\mathrm{OH}^{-}, \mathrm{Pd}^{0}$ 作为路易斯酸活化腈, 之后 $\mathrm{OH}^{-}$对腈亲核进攻形成 $\mathrm{C}-$ $\mathrm{O}$ 键; DFT 计算表明 $\mathrm{C}-\mathrm{O}$ 键形成过程是反应的决速步 骤, 所需的活化能最高, 且在该过程中 $\mathrm{N}-\mathrm{Pd}$ 键之间的 距离从 $0.2138 \mathrm{~nm}$ 变为 $0.2008 \mathrm{~nm}$, 这说明 $\mathrm{Pd}$ 原子通过 接受氰基中氮原子的负电荷促进亲核进攻过程; 之后 $\mathrm{Pd}$ 表面的 $\mathrm{OH}^{-}$与带负电荷的氮原子之间因存在库伦作 用得到中间体 $\mathbf{8 8}$, 氮原子进而从 $\mathrm{OH}^{-}$中获得质子得到 89, 最后在溶剂中得到产物酰胺(Scheme 14).

Ag NP 价格相对低廉, 容易合成, 被广泛应用于催 


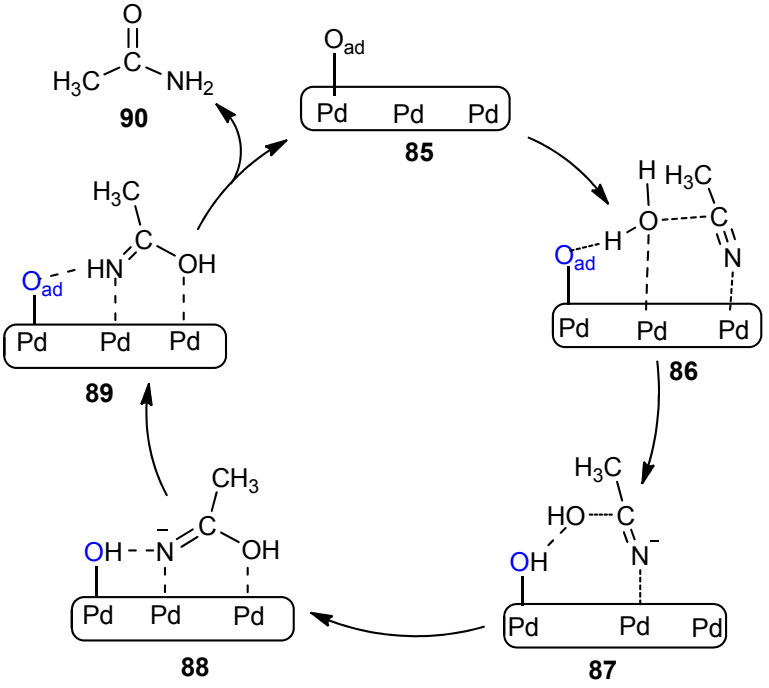

图式 $14 P d$ NPs 催化的腈的水合反应机理

Scheme 14 Pd NPs-catalyzed reaction mechanism for hydration reactions of nitriles

化腈的水合反应. 2014 年, Sherbow 等 ${ }^{[53]}$ 用 1,3,5-三氮-7磷酸金刚烷(PTA)作为配体稳定 AgNP 得到 Ag-PTA NP 91, 并用于催化腈的水合反应. 该催化剂是 $\mathrm{AgNO}_{3}$ 与 $\mathrm{NaBH}_{4}$ 在冷水中还原制备出 $\mathrm{Ag} \mathrm{NP}$ 后, 加入水溶性的 PTA 配体后制备而得的橙色纳米颗粒. 研究表明, 当不 加入 PTA 配体时, Ag NP 通过吸附硼氢化物达到稳定 ${ }^{[54]}$, 但一段时间后, 硼氢化物分解, Ag NP 会聚集成大块金 属沉淀下来, 而 PTA 配体上的 N、P 原子与 $\mathrm{Ag}$ 配位得 到的稳定性能更强的 91, 且有效避免 $\mathrm{Ag} N \mathrm{NP}$ 以较大颗 粒聚集. 在催化腈水合反应的过程中, 91 可在 $90{ }^{\circ} \mathrm{C}$ 、空 气气氛下使芳基腈和部分杂芳基腈转化为相应的酰胺. 当催化 $\alpha$-羟基腈水合反应时, 催化剂由原来的橙色变为 无色, 溶解在溶液中, 反应一段时间后 $\alpha$-羟基腈部分转 化成酰胺, 向反应后的溶液继续加入 $\alpha$-羟基腈后, $\alpha$-羟 基腈仍能转化成酰胺, 说明在 $\mathrm{HCN}$ 存在的条件下溶液 仍具有催化活性. 通过控制实验证明, 91 在含有 $\mathrm{HCN}$ 的 溶液中生成了 $\operatorname{Ag}(\mathrm{CN})_{n}{ }^{1-n}$ 型配合物 92, 在反应的初始阶 段, 随着 $\mathrm{HCN}$ 含量的增加, 92 含量也增加, 使得催化效 率提升. 由于该配合物金属中心不稳定，有效避免了催 化剂在反应过程被毒害, 故在 $\mathrm{HCN}$ 存在的条件下保持 催化活性, 且该配合物中 $\mathrm{CN}^{-}$的吸电子能力较强, 其催 化活性较佳. 这种具有不稳定电子构型催化剂, 一定程 度上缓解了均相体系中金属催化剂被毒害的问题, 为 $\alpha-$ 羟基腈水合催化剂的设计提供新的思路.

金纳米材料催化剂因能促进低温反应的选择性而 备受关注, 但其在腈水合反应领域鲜有报道. 2012 年, 曹勇等 ${ }^{[5]}$ 以 $\mathrm{TiO}_{2}$ 为载体制备 $\mathrm{Au} / \mathrm{TiO}_{2} 93$, 该催化剂是金 首次作为非均相催化剂用于催化腈的水合反应, 在反应 过程中, 催化剂的金属表面和 $\mathrm{TiO}_{2}$ 分别活化腈和水分
子，再经过水分子的亲核进攻得到产物. 2016 年, Kumar 等 ${ }^{[56]}$ 用聚苯乙烯负载的金催化剂 Au/PS NP 94 在微波辐 射的辅助下在较低的反应温度、较短的反应时间内使腈 高效地水合成酰胺，该反应底物范围广，含多种取代基 的芳基腈及杂芳基腈均能较好地参与反应，且在不降低 催化效率的条件下催化剂可回收使用 8 次.

除了将金属单质负载到载体上或添加配体达到稳 定状态，金属氧化物纳米颗粒也可用于催化腈的水合反 应. 2017 年, Mehta 等 ${ }^{[57]}$ 用表面活性剂法制备了 $\mathrm{MnO}_{2}$ 纳 米材料, 并将其作为光催化剂, 在可见光的条件下于中 性或碱性溶液中催化腈的水合反应. 该催化剂的比表面 积大, 催化活性位点多, 因而催化效果较好, 且催化剂 的表面将水分子分散开来, 避免了酰胺进一步水解成羧 酸. 当添加 $\mathrm{NEt}_{3}$ 时, 反应体系呈碱性, 亲核能力较强的 $\mathrm{OH}^{-}$对氧基中的碳原子进行亲核进攻，使反应时间大大 缩短 (Scheme 15).
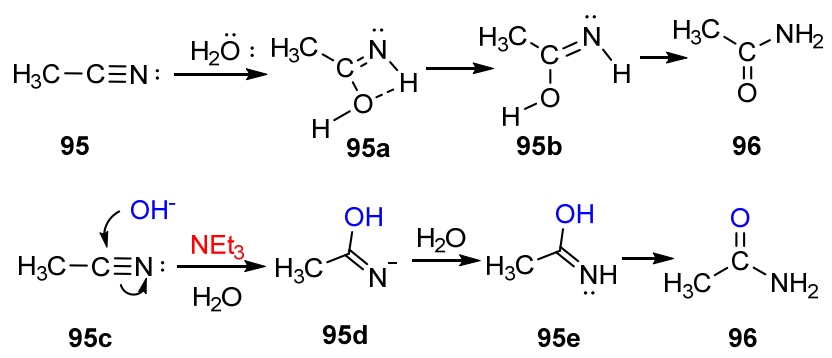

图式 15 在中性和碱性条件下腈的水合反应机理

Scheme 15 Reaction mechanisms for hydration reactions of nitriles in neutral and alkaline conditions

2019 年, 肖丰收等 ${ }^{[58]}$ 用 $\mathrm{MnO}_{2}$ 纳米棒 $\mathrm{NR}-\mathrm{MnO}_{2} 97$ 催化腈的水合反应. 将反应物与催化剂置于高压釜中, 在 $75{ }^{\circ} \mathrm{C}$ 下反应 $2 \mathrm{~h}$, 芳基腈、杂芳基腈均能高效地转化 为相应的酰胺, 其转化率和选择性均高于 $99.5 \%$, 催化 效果甚至优于均相催化剂, 对于部分烷基腈如己腈和癸 腈的催化效果也较佳. 在反应过程中，催化剂上的氧活 性位点通过与吸附在表面的水分子形成 $\mathrm{Mn} \cdots \mathrm{O} \cdots \mathrm{H}-$ $\mathrm{OH}$ 键激活水分子生成羟基得到中间体 101, 羟基进而 对氧基进行亲核进攻得到产物酰胺(Scheme 16).

金属纳米催化剂的设计通常将金属纳米颗粒负载 到其它载体上、添加配体达到稳定或直接形成金属氧化 物纳米颗粒, 在反应过程中, 金属纳米催化剂将腈和水 分子吸附在其表面，利用金属中心、配体或载体活化水 分子生成 $\mathrm{OH}^{-}$促进亲核进攻过程. 与高价态金属催化 剂相比, 金属纳米催化剂的金属中心可能由于电子密度 增加，导致与反应底物的结合能力较弱，但这也避免了 催化剂在反应过程中失活; 且金属纳米催化剂比表面积 高, 催化活性位点多, 因此催化效果较佳, 反应结束后 容易从体系中分离并再利用，由此可见金属纳米催化剂 
在腈水合反应领域存在较高的研究价值.

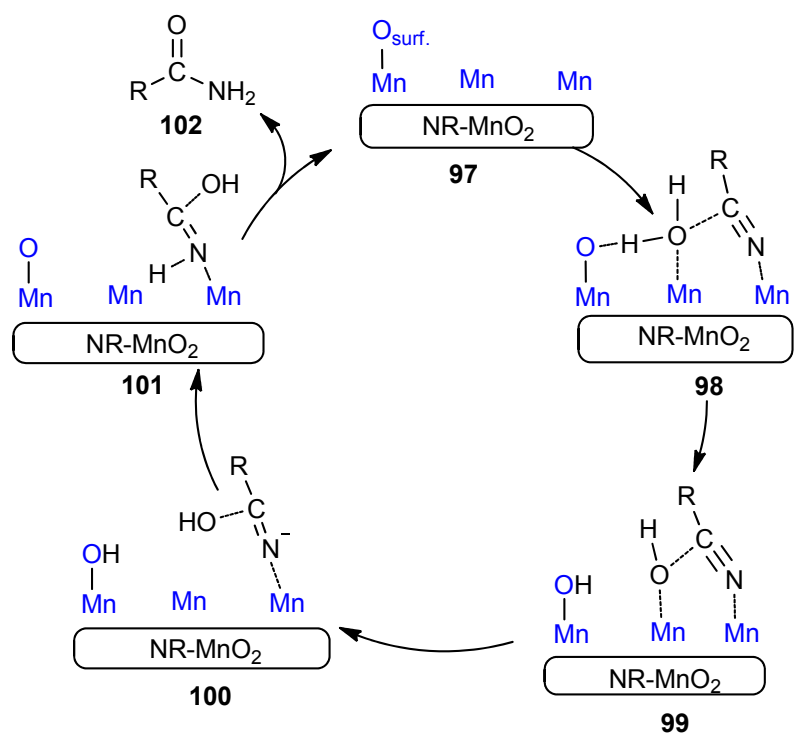

图式 $16 \mathrm{NR}-\mathrm{MnO}_{2}$ 催化的腈的水合反应机理

Scheme 16 NR- $\mathrm{MnO}_{2}$-catalyzed reaction mechanism for hydration reactions of nitriles

\section{4 离子液体催化腈的水合反应}

离子液体指在室温条件下呈液态, 完全由阴阳离子 组成的有机试剂[ ${ }^{[59-61]}$. 离子液体溶解能力强, 可溶解有 机物、无机物及有机金属化合物等, 此外, 其蒸汽压基 本为零, 并且容易回收, 可循环使用 ${ }^{[62-64]}$. 在反应中, 离子液体可作为催化剂、溶剂和有机试剂，引起了研究 工作者极大的兴趣, 并将其作为催化剂催化腈的水合反 应. 在离子液体的催化下, 腈也可较好地转化为酰胺, 且减少了有毒有害物质的使用.

2011 年, Kalkhambkar 等 ${ }^{[65]}$ 以 $\left[\mathrm{BMIM}\left(\mathrm{SO}_{3} \mathrm{H}\right)\right][\mathrm{OTf}]$ 103 作为催化剂, 催化腈与叔醇发生 Ritter 反应得到酰 胺. 作者分别用叔丁醇、金刚烷醇和甲基环戊醇作为碳 正离子源与部分烷基腈、芳基腈及杂芳基腈反应，均能 在相对温和的条件下得到较高的产率. 2014 年, Kumar 等 ${ }^{[66]}$ 用离子液体 $[\mathrm{bmim}] \mathrm{HSO}_{4}$ (104)催化腈转化为羧酸. 该离子液体中含有一部分水, 可与催化剂相互作用, 活 化腈后，作为亲核试剂对氧基进行亲核进攻得到酰胺， 酰胺最终在催化剂和水的作用下进一步水解成羒酸. 该 体系具有反应时间短、条件温和、效率高且底物适应性 广等优点. 2015 年, Veisi 等 ${ }^{\left[{ }^{[7]}\right.}$ 以四丁基氢氧化铵(TBAH) 105 作为催化剂, 乙醇为溶剂, 在不添加金属的条件下 催化腈反应生成酰胺. 反应过程中，105 与㲵基配位从 而活化腈, 然后受到相邻羟基离子的亲核进攻后生成酰 胺(Scheme 17). 该反应可有效催化芳香腈、烷基腈和杂 环腈水合为相应的酰胺, 并避免其过度水解为羧酸, 且 在该催化条件下，当底物含有两个㲵基时，可通过控制
反应时间选择性得到单个氧基水解或两个氧基均水解 的产物.

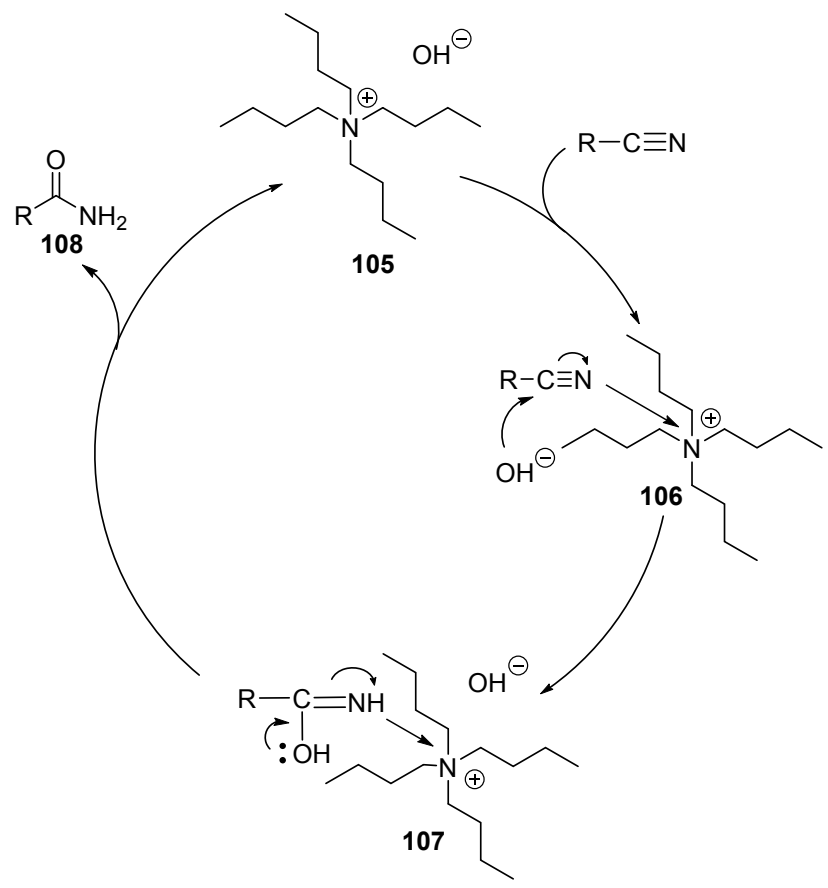

图式 17 TBAH 催化的腈的水合反应机理

Scheme 17 TBAH-catalyzed reaction mechanism for hydration reactions of nitriles

2020 年, Dutta 等 $^{[68]}$ 用 $[\mathrm{Nbdm}] \mathrm{OH} 109$ 作为溶剂和催 化剂催化 2-氨基苯腈和醛或酮在室温条件下反应生成 2,3-二氢喹唑啉-4 $(1 H)$ 类化合物. 109 作为双核离子液体 与单核离子液体相比,有更多的活性位点, 彼此的协同 作用较好且有更强的稳定性，有利于提高催化剂的催化 性能. 在该反应中, 109 中的正负离子中心分别于两分 子的酮或醛、两分子的 2-氨基苯腈作用，生成两个席夫 碱中间体 114，之后 109 上的两个 $\mathrm{OH}^{-}$分别对其进行亲 核进攻得到酰胺，酰胺上氮原子进一步对 114 上的碳原 子进行亲核进攻，发生环化反应，最终得到产物分子 (Scheme 18).

离子液体特有的物化性质，使其在有机反应中占有 独特的优势，同时可通过改变阴阳离子的种类改变离子 液体的性质. 在作为催化剂促进腈的水合反应过程中, 催化机制多为阳离子中心与腈配位后完成对腈的活化, 阴离子如 $\mathrm{OH}^{-}$再对腈进行亲核进攻，以此促进反应的 完成.

\section{5 其它条件下腈的水合反应}

随着对该领域研究的不断深入, 其它类型的催化体 系也相继被开发出来，腈水合反应的条件逐渐得以优化 和完善. 2012 年, 涂涛等 ${ }^{[69]}$ 在不添加金属、有机配体及 有机溶剂的条件下，用一系列无机盐如 $\mathrm{CaCO}_{3} 、 \mathrm{Cs}_{2} \mathrm{CO}_{3}$ 、 


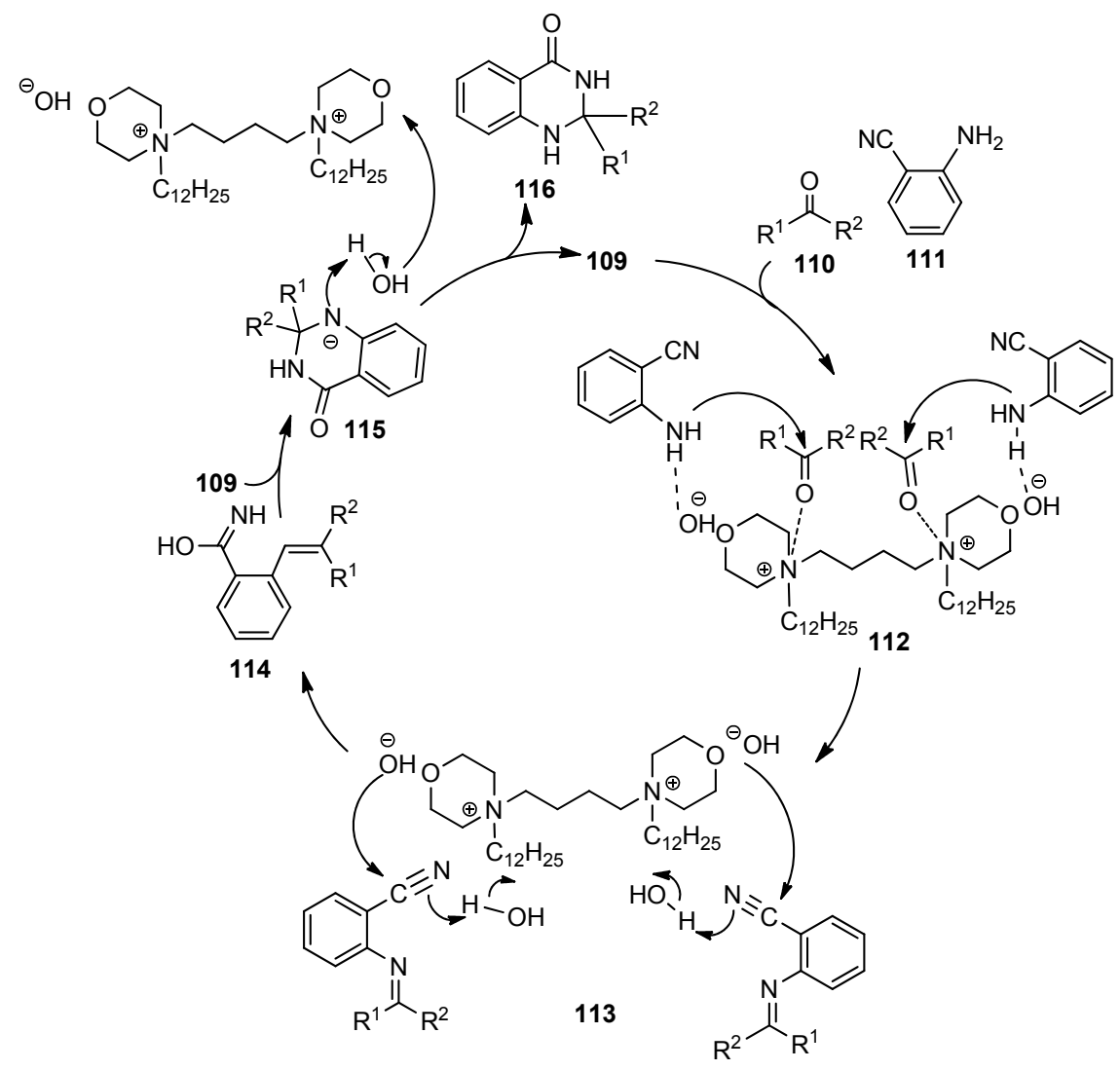

图式 $18[\mathrm{Nbdm}] \mathrm{OH}$ 催化的腈的水合反应机理

Scheme 18 [Nbdm] OH-catalyzed reaction mechanism for hydration reactions of nitriles

$\mathrm{K}_{2} \mathrm{CO}_{3} 、 \mathrm{KOAc} 、 \mathrm{~K}_{3} \mathrm{PO}_{4}$ 等在水溶液中催化腈的水合反应 (Eq. 6). 经过条件篮选, 作者以 $\mathrm{K}_{2} \mathrm{CO}_{3}$ 为催化剂(5 50 $\mathrm{mol} \%$ ), 在 $150{ }^{\circ} \mathrm{C}$ 的反应温度下, 利用微波辐射加快反 应进行, 使多数腈在 15 40 min 内转化为酰胺. 在该催 化体系下, 含有吸电子基团的芳基腈在较短的时间内高 效地转化为酰胺, 且催化剂添加量仅需 $5 \mathrm{~mol} \%$, 而含有 供电子基团的芳基腈、空间位阻较大的腈及部分杂芳基 腈、烷基腈, 通过增加催化剂的使用量和延长反应时间 也能较好地完成转化. 将反应后的滤液循环使用三次 后, 溶液的 $\mathrm{pH}$ 值显著降低, 说明 $\mathrm{OH}^{-}$在反应过程中起 重要作用. 因此, 该反应过程中, 水分子中的羟基在 $\mathrm{K}_{2} \mathrm{CO}_{3}$ 的作用下获得一个电子, 随后对氧基进行亲核进 攻, 最终在水的作用下得到产物酰胺(Scheme 19).

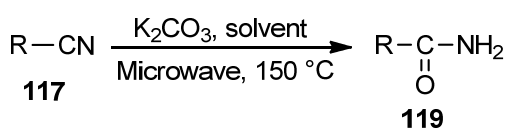

在以往的报道中, 金属氢氧化物在体系中作为催化 剂, 二甲基亚砜(DMSO)作为溶剂, 而 $\mathrm{M}_{\mathrm{A}} \mathrm{OH}\left(\mathrm{M}_{\mathrm{A}}=\mathrm{Cs}\right.$, $\mathrm{Na}, \mathrm{K}$ 等)和 DMSO 作为催化剂在有机反应中的报道较 少. 2014 年, 徐清等 ${ }^{[70]}$ 用 $\mathrm{CsOH-DMSO}$ 作为催化剂在 $100{ }^{\circ} \mathrm{C}$ 以内的条件下将腈水合成酰胺(Eq. 7), 在该反应

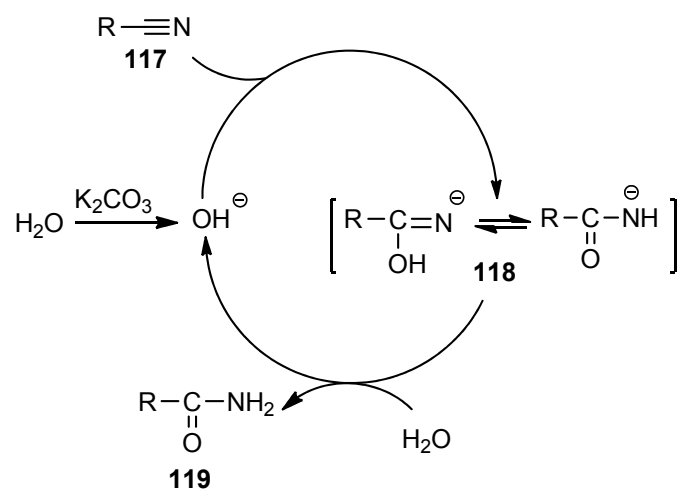

图式 $19 \mathrm{~K}_{2} \mathrm{CO}_{3}$ 催化的腈的水合反应机理

Scheme $19 \mathrm{~K}_{2} \mathrm{CO}_{3}$-catalyzed reaction mechanism for hydration reactions of nitriles

中加入较多 DMSO 降低体系中的 $\mathrm{OH}^{-}$, 从而抑制酰胺 进一步水解成羧酸. 该反应中, 作者提出两种反应路径 (Scheme 20), $\mathrm{CsOH}$ 首先与 DMSO 反应生成二甲基亚砜 阴离子 $\mathrm{S}$ 和 $\mathrm{Cs}^{+}, \mathrm{Cs}^{+}$与氧基配位后与 $\mathbf{1 2 2}$ 反应得到中间 体 123. 在路径(a)中, 123 直接在水的进攻下水解成酰 胺; 在路径(b)中, 水进攻 DMSO 部分的硫中心得到产 物酰胺. 该催化条件下底物适用性较广, 含吸电子基团 或供电子基团的芳基腈、杂环腈和部分烷基腈都能有效 
转化. 值得注意的是, 芳基腈和烷基腈转化时间长达 30 $\mathrm{h}$, 而杂环腈在 $3 \mathrm{~h}$ 内即能完成转化. 作者推测可能是杂 环上的氮或硫原子对氧基有着一定的活化作用，从而使 杂环腈能在较短的时间内完成转化.
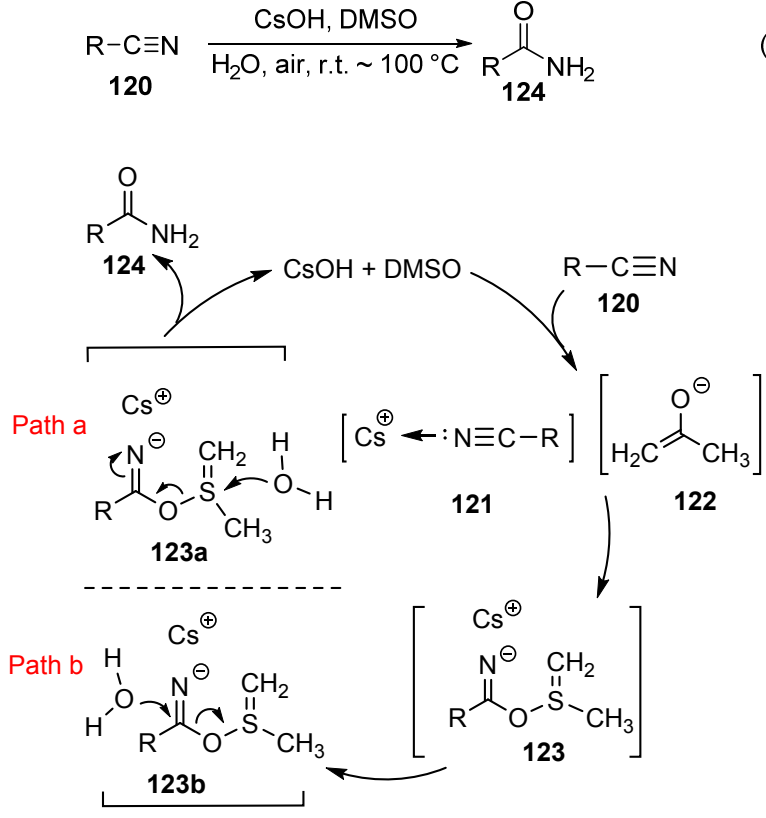

图式 20 CsOH-DMSO 催化的腈的水合反应机理

Scheme 20 CsOH-DMSO-catalyzed reaction mechanism for hydration reactions of nitriles

2015 年, Midya 等 ${ }^{[71]}$ 将 $\mathrm{KO}^{t} \mathrm{Bu}$ 作为氧源和催化剂在 室温条件下催化腈的水合反应, 在该催化体系下不需要 添加过渡金属催化剂，且能有效抑制酰胺过度水解成羧 酸. 反应过程中, 腈在 $\mathrm{KO}^{t} \mathrm{Bu}$ 的作用下通过阳离子 $-\pi$ 相 互作用生成配合物 126, 阳离子配合物 126 与叔丁基阴 离子之间经过单电子转移形成 127 ; 叔丁基中氧原子进 攻氰基中的碳正原子生成六元环中间体 128，随后脱去 一分子 2-甲基丙烯, 最后经过质子化得到产物酰胺 (Scheme 21). 在底物拓展过程中, 作者发现当以甲苯为 溶剂时, 芳基腈邻位存在取代基如 $\mathrm{F} 、 \mathrm{Cl}$ 及 $\mathrm{Br}$ 等，由于 取代基与六元环中间体之间存在空间位阻效应，导致生 成中间体 128a 需要更多的活化能, 且 128a 由于邻位取 代基与甲基存在相互作用, 因而不稳定, 于是将反应温 度升高到 $60{ }^{\circ} \mathrm{C}$ 、反应时间延长至 $12 \mathrm{~h}$ 才能得到相应的 酰胺; 而以 ${ }^{t} \mathrm{BuOH}$ 为溶剂时, 芳基腈的邻位或对位取代 基为 $\mathrm{F} 、 \mathrm{Cl}$ 和 $\mathrm{Br}$ 时, 与 ${ }^{t} \mathrm{BuOH}$ 生成氢键, 减弱了取代基 与六元环之间的相互作用, 增加了构象的空间体积, 使 反应过程不需要经历能量较高的过渡态, 在室温条件下 便能得到相应的产物(Scheme 22).

近年来, 虽然研究人员开发了不同类型的催化剂使 腈在相对温和的条件下实现了高选择性的水合反应. 而 $\alpha$-氨基腈仍然需要比较苛刻的反应条件, 如强酸及高
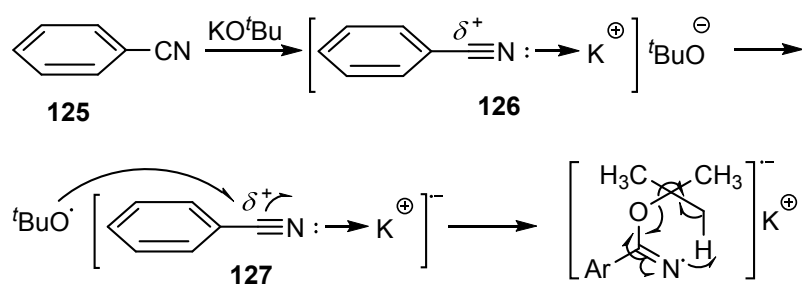

128
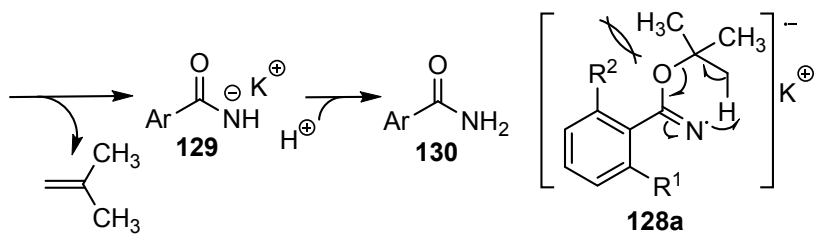

图式 $21 \mathrm{KO}^{t} \mathrm{Bu}$ 催化的腈的水合反应机理 Scheme $21 \mathrm{KO}^{t} \mathrm{Bu}$-catalyzed reaction mechanism for hydration reactions of nitriles
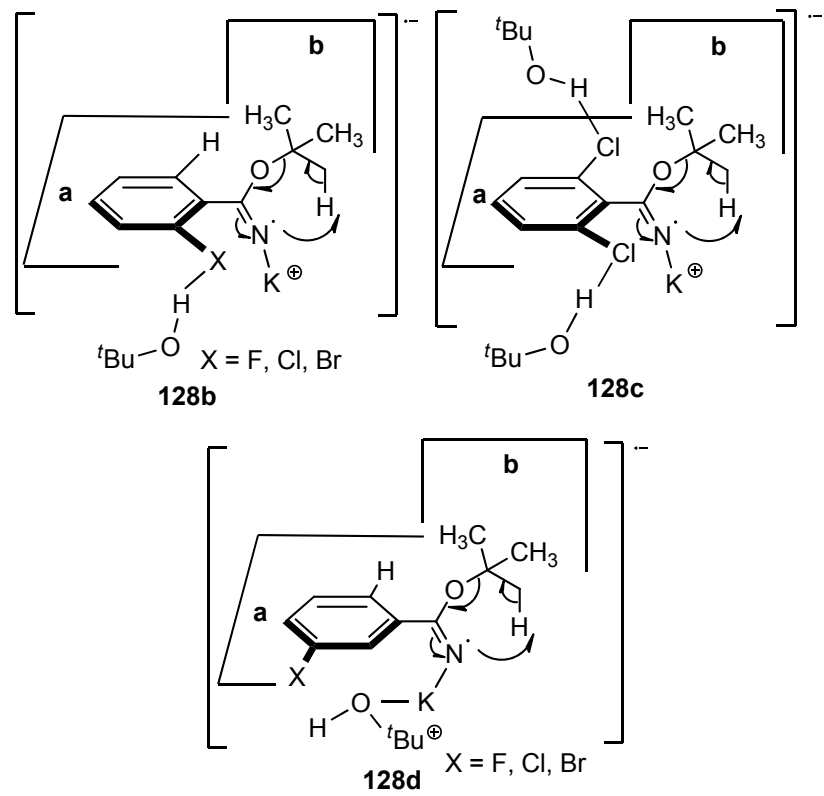

图式 22 不同底物的溶剂效应

Scheme 22 Solvent effect of different substrates

温, 且反应时间较长. Sola 等 ${ }^{[72]}$ 曾用丙酮作为催化剂, 在添加 $\mathrm{NaOH}$ 的条件下经过分子内反应得到相应的酰 胺(Eq. 8), 虽然该催化条件下催化效果较好，但催化剂 和碱的用量较大, 分别高达 5 和 10 equiv. 为了减少催化 剂和碱的添加量, 2016 年, Chitale 等 ${ }^{[72]}$ 篮选了不同的羰 基化合物，最终发现添加 $20 \mathrm{~mol} \% \mathrm{NaOH}$ 时，在 20 $\mathrm{mol} \%$ 甲醛的催化下，含不同取代基的 $\alpha$-氨基腈可在室 温条件下高效地完成水合反应(Eq. 9). 当氨基上含有一 个取代基形成二级胺时，甲醛依旧保持较高的催化活 性，且添加对映体的底物在反应过程中构型不会发生变 化, ee 值高达 $97 \%$ ，说明手性醛类化合物在反应中可作 为一类高效的不对称催化剂. $\alpha$-氨基腈在 $\mathrm{HCHO}$ 和 $\mathrm{NaOH}$ 的作用下生成中间体 $\mathbf{1 3 5}$, 再经过分子内亲核进 
攻得到产物酰胺.

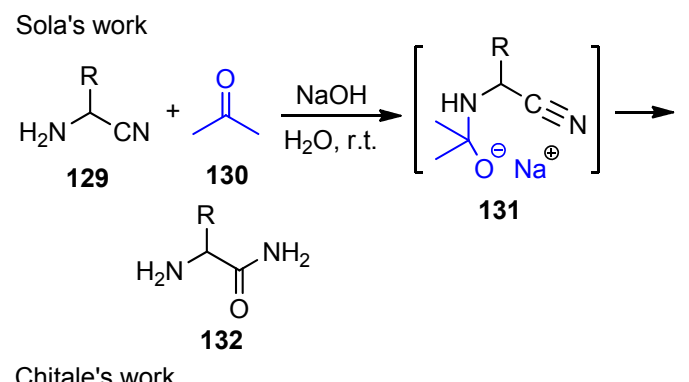

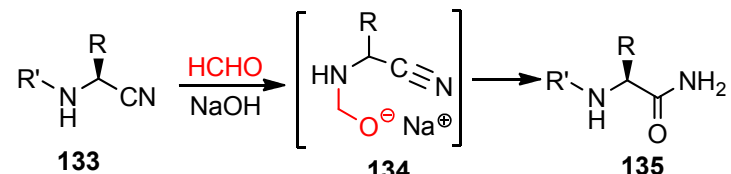

\section{6 总结与展望}

主要介绍了近年来芳基腈、杂芳基腈和烷基腈在不 同催化体系下水合反应生成酰胺的研究现状. 不同的催 化体系如过渡金属配合物催化体系、金属阳离子催化体 系、金属纳米粒子催化体系、离子液体催化体系及其它 类型催化体系已被开发. 这些催化体系不同程度上优化 了腈水合反应的条件, 如减少催化剂的使用量、反应时 间缩短至 $1 \mathrm{~h}$ 甚至 $10 \mathrm{~min}$ 及产率提高至 $99 \%$ 等. 在腈水 合反应整个历程中，不同的催化剂催化机理虽然不同， 但大多通过两个方面来促进反应的进行：(1)与氰基作 用, 活化氰基, 使其更容易参与反应; (2)增强亲核试剂 的亲核性, 促进亲核进攻过程. 如金属配合物催化剂的 金属中心与㲵基配位后，催化剂配体与水分子作用生成 氢键等增强水分子中氧原子的亲核性; 而金属钠米粒子 催化剂可以通过表面氧原子使水分子解离成亲核能力 更强的羟基来促进反应. 这些催化体系的开发与不断发 展, 为腈的水合反应提供了丰富有效的策略. 尽管腈的 水合反应研究取得了显著的进展, 但仍存在许多未解决 的问题和挑战. (1)大多数催化体系涉及贵金属如钯、钉、 铑、铂以及有机溶剂的使用, 反应后催化剂难以回收和 重复利用, 成本与环境污染等问题仍有待改善. (2)底物 范围较为受限, 如在很多反应中吸电子基团或空间位阻 较大的腈作底物时, 其相应酰胺产率略低, 杂芳基腈及 烷基腈也因不能较好地生成中间体导致转化率低. (3) $\alpha$ 羟基腈的水合作用一直是该领域的难点, 尽管某些催化 剂可以转化部分 $\alpha$-着基腈, 但也大多针对醛衍生的 $\alpha$-差弪 基腈有效, 而酮衍生的 $\alpha$-羟基腈由于稳定性较差, 更易 分解成相应的酮和 $\mathrm{HCN}$, 因而很难经过水合反应生成 酰胺; 其次 $\alpha$-差基腈发生副反应得到的 $\mathrm{HCN}$ 会毒化催 化剂, 因而使 $\alpha$-羟基腈的水合反应更难进行. 面对腈水 合反应中存在的催化剂毒化问题, 应广泛探索与开发光
化学及电化学等新兴的可持续合成手段, 为腈的水合反 应尤其是酮衍生的 $\alpha$-差基腈的高效水合提供了建设性 参考. 总之, 近年来研究工作者对腈的水合反应已有了 较为深入的研究, 但仍面临许多挑战, 面对这些难点, 进一步探索更优、更为普遍的催化体系成为后续研究工 作者关注的焦点和难点.

\section{References}

[1] Song, C.-Y.; Qu, S.-L; Tao, Y.; Dang, Y.-F; Wang, Z.-X. ACS Catal. 2014, 4, 2854.

[2] Pattabiraman, V. R.; Bode, J. W. Nature 2011, 480, 471

[3] Gunanathan, C.; Ben-David, Y.; Milstein, D. Science 2007, 317, 790.

[4] Mahjour, B.; Shen, Y.; Liu, W.; Cernak, T. Nature 2020, 580, 71.

[5] García-Álvarez, R.; Francos, J.; Tomás-Mendivil, E.; Crochet, P.; Cadierno, V. J. Org. Chem. 2014, 79, 93.

[6] Wang, Y.; Chen, C.; Zhang, S.; Lou, Z.-B.; Su, X.; Wen, L.-R.; Li, M. Org. Lett. 2013, 15, 4794 .

[7] Ma, B.; Wang, Y.; Peng, J.; Zhu, Q. J. Org. Chem. 2011, 76, 6362.

[8] Wu, Z.; Ren, R.; Zhu, C. Angew. Chem., Int. Ed. 2016, 55, 10821.

[9] Tílvez, E.; Menéndez, M. I.; López, R. Organometallics 2012, 31, 1618.

[10] Lee, J.; Kim, M.; Chang, S.; Lee, H. Y. Org. Lett. 2009, 11, 5598.

[11] Guo, B.; de Vries, J. G.; Otten, E. Chem. Sci. 2019, 10, 10647.

[12] Ahmed, T. J.; Knapp, S. M. M.; Tyler, D. R. Coord. Chem. Rev. 2011, 255, 949.

[13] Kim, J. H. Angew. Chem., Int. Ed. 1990, 29, 523

[14] Diomand, S. E.; Grant, B.; Tom, G. M. Tetrahedron Lett. 1974, 15, 4025.

[15] Murahashi, S. I.; Naota, T. Bull. Agric. Chem. Soc. Jpn. 1996, 69, 1805.

[16] García-Álvarez, R.; Crochet, P.; Cadierno, V. Green Chem. 2013, 15,46 .

[17] Fung, W. K.; Huang, X.; Man, M. L.; Ng, S. M.; Hung, M. Y.; Lin, Z.; Lau, C. P. J. Am.Chem. Soc. 2003, 125, 11539.

[18] Cadierno, V.; Diez, J.; Francos, J.; Gimeno, J. Chem.-Eur. J. 2010 , 16,9808 .

[19] García-Álvarez, R.; Díez, J.; Crochet, P.; Cadierno, V. Organometallics 2011, 30, 5442.

[20] Knapp, S. M. M.; Sherbow, T. J.; Yelle, R. B.; Zakharov, L. N.; Juliette, J. J.; Tyler, D. R. Organometallics 2013, 32, 824.

[21] Tomás-Mendivil, E.; García-Álvarez, R.; Vidal, C.; Crochet, P.; Cadierno, V. ACS Catal. 2014, 4, 1901.

[22] Tomás-Mendivil, E.; Menéndez-Rodríguez, L.; Francos, J.; Crochet, P.; Cadierno, V. RSC Adv. 2014, 4, 63466.

[23] Geldbach, T. J.; Drago, D.; Pregosin, P. S. Chem. Commun. 2000 1629.

[24] Geldbach, T. J.; Breher, F.; Gramlich, V.; Kumar, P. G. A.; Pregosin, P. S. Inorg. Chem. 2004, 43, 1920.

[25] Leung, C. W.; Zheng, W. X.; Wang, D. X.; Ng, S. M.; Yeung, C. H.; Zhou, Z. Y.; Lin, Z. Y.; Lau, C. P. Organometallics 2007, 26, 1924.

[26] Tomás-Mendivil, E.; Suárez, F. J.; Díez, J.; Cadierno, V. Chem. Commun. 2014, 50, 9661.

[27] Ghaffar, T.; Parkins, A. W. Tetrahedron Lett. 1995, 36, 8657.

[28] Xing, X.-Y.; Xu, C.; Chen, B.; Li, C.-C.; Virgil, S. C.; Grubbs, R. H. J. Am. Chem. Soc. 2018, 140, 17782.

[29] Paul, B.; Maji, M.; Kundu, S. ACS Catal. 2019, 9, 10469.

[30] Breno, K. L.; Pluth, M. D.; Tyler, D. R. Organometallics 2003, 22, 1203.

[31] Takaya, H.; Yoshida, K.; Isozaki, K.; Terai, H.; Murahashi, S. Angew. Chem., Int. Ed. 2003, 42, 3302.

[32] Goto, A.; Endo, K.; Saito, S. Angew. Chem., Int. Ed. 2008, 47, 3607.

[33] Anderson, N. H.; Boncella, J. M.; Tondreau, A. M. Organometallics 
2018, 37, 4675

[34] Kim, E. S.; Kim, H. S.; Kim, J. N. Tetrahedron Lett. 2009, 50, 2973.

[35] Kim, E. S.; Lee, H. S.; Kim, S. H.; Kim, J. N. Tetrahedron Lett. 2010, 51,1589

[36] Ma, X.-Y.; He, Y.; Hu, Y.-L.; Lu, M. Tetrahedron Lett. 2012, 53, 449.

[37] Ma, X.-Y.; He, Y.; Wang, P.-C.; Lu, M. Appl. Organomet. Chem. 2012, 26, 377 .

[38] Ma, X.-Y.; He, Y.; Lu, M. Synth. Commun. 2013, 44, 474.

[39] Sanz Sharley, D. D.; Williams, J. M. J. Tetrahedron Lett. 2017, 58, 4090.

[40] Kanda, T.; Naraoka, A.; Naka, H. J. Am. Chem. Soc. 2019, 141, 825 .

[41] Oberhauser, W.; Bartoli, M.; Petrucci, G.; Bandelli, D. J. Mol. Catal. A: Chem. 2015, 410, 26.

[42] Mitsudome, T.; Mikami, Y.; Mori, H.; Arita, S.; Mizugaki, T.; Jitsukawa, K.; Kaneda, K. Chem. Commun. 2009, 3258.

[43] Woo, H.; Lee, K.; Park, S.; Park, K. H. Molecules 2014, 19, 699.

[44] Kim, A. Y.; Bae, H. S.; Park, S.; Park, S.; Park, K. H. Catal. Lett. 2011, 141,685 .

[45] Shimizu, K.-I.; Imaiida, N.; Sawabe, K.; Satsuma, A. Appl. Catal., A. 2012, 421 422, 114.

[46] Gangarajula, Y.; Gopal, B. Appl. Catal., A 2014, 475, 211.

[47] Baig, R. B.; Varma, R. S. Chem. Commun. 2012, 48, 6220.

[48] Yan, N.; Xiao, C.-X.; Kou, Y. Coord. Chem. Rev. 2010, 254, 1179.

[49] Gong, J. L.; Mullins, C. B. Acc. Chem. Res. 2009, 42, 1063.

[50] Gladys, M. J.; El Zein, A. A.; Mikkelsen, A.; Andersen, J. N.; Held, G. Surf. Sci. 2008, 602, 3540.

[51] Pan, M.; Hoang, S.; Mullins, C. B. Catal. Today 2011, 160, 198.

[52] Shimizu, K.-I.; Kubo, T.; Satsuma, A.; Kamachi, T.; Yoshizawa, K. ACS Catal. 2012, 2, 2467.

[53] Sherbow, T. J.; Downs, E. L.; Sayler, R. I.; Razink, J. J.; Juliette, J. J.; Tyler, D. R. ACS Catal. 2014, 4, 3096.

[54] Mulfinger, L.; Solomon, S. D.; Bahadory, M.; Jeyarajasingam, A.
V.; Rutkowsky, S. A.; Boritz, C. J. Chem. Educ. 2007, 84, 322.

[55] Liu, Y.-M.; He, L.; Wang, M.-M.; Cao, Y.; He, H.-Y.; Fan, K.-N. ChemSusChem 2012, 5, 1392.

[56] Kumar, S.; Sharma, S.; Das, P. Adv. Synth. Catal. 2016, 358, 2889.

[57] Mehta, A.; Basu, S. J. Photochem. Photobiol., A 2017, 343, 1.

[58] Wang, H.; Wang, Y.-Q.; Xu, H.; Zhou, H.; Wang, L.; Meng, X.-J.; Xiao, F.-S. Ind. Eng. Chem. Res. 2019, 58, 17319

[59] Goossens, K.; Lava, K.; Bielawski, C. W.; Binnemans, K. Chem. Rev. 2016, 116, 4643.

[60] Rantwijk, F. V.; Sheldon, R. A. Chem. Rev. 2007, 107, 2757.

[61] Han, X. X.; Armstrong, D. W. Acc. Chem. Res. 2007, 40, 1079.

[62] Veisi, H.; Manesh, A. A.; Khankhani, N.; Ghorbani-Vaghei, R. RSC Adv. 2014, 4, 25057.

[63] Earle, M. J.; Esperanca, J. M.; Gilea, M. A.; Lopes, J. N.; Rebelo, L. P.; Magee, J. W.; Seddon, K. R.; Widegren, J. A. Nature 2006 $439,831$.

[64] Dong, K.; Liu, X.-M.; Dong, H.-F.; Zhang, X.-P.; Zhang, S.-J. Chem. Rev. 2017, 117, 6636

[65] Kalkhambkar, R. G.; Waters, S. N.; Laali, K. K. Tetrahedron Lett. 2011, 52, 867

[66] Kumar, S.; Dixit, S. K.; Awasthi, S. K. Tetrahedron Lett. 2014, 55, 3802.

[67] Veisi, H.; Maleki, B.; Hamelian, M.; Ashrafi, S. S. RSC Adv. 2015, 5,6365 .

[68] Dutta, A.; Damarla, K.; Kumar, A.; Saikia, P. J.; Sarma, D. Tetrahedron Lett. 2020, 61, 151587.

[69] Tu, T.; Wang, Z.-X.; Liu, Z. L.; Feng, X.-K.; Wang, Q.-Y. Green Chem. 2012, 14, 921

[70] Chen, H.-N.; Dai, W.-J.; Chen, Y.; Xu, Q.; Chen, J.-H.; Yu, L.; Zhao, Y.-J.; Ye, M.-D.; Pan, Y.-J. Green Chem. 2014, 16, 2136.

[71] Midya, G. C.; Kapat, A.; Maiti, S.; Dash, J. J. Org. Chem. 2015, 80, 4148.

[72] Chitale, S.; Derasp, J. S.; Hussain, B.; Tanveer, K.; Beauchemin, A. M. Chem. Commun. 2016, 52, 13147.

(Cheng, F.) 